Draft Version OCtober 10, 2018

Preprint typeset using $\mathrm{IATEX}_{\mathrm{E}}$ style emulateapj v. 12/16/11

\title{
THE METAL-POOR NON-SAGITTARIUS (?) GLOBULAR CLUSTER NGC 5053: ORBIT AND MG, AL AND SI ABUNDANCES
}

\author{
Baitian Tang ${ }^{1,2}$, J. G. Fernández-Trincado ${ }^{2,10}$, Doug Geisler ${ }^{2}$, Olga Zamora ${ }^{3,4}$, Szabolcs Mészáros ${ }^{5,17}$, Thomas \\ Masseron $^{3,4}$, Roger E. Cohen ${ }^{6}$, D. A. García-Hernández ${ }^{3,4}$, Flavia Dell'Agli ${ }^{3,4}$, Timothy C. Beers ${ }^{7}$, Ricardo P. \\ Schiavon $^{8}$, Sangmo Tony Sohn ${ }^{6}$, Sten Hasselquist ${ }^{9}$, Annie C. Robin ${ }^{10}$, Matthew Shetrone ${ }^{11}$, Steven R. \\ Majewsit $^{12}$, Sandro Villanova ${ }^{2}$, Jose Schiappacasse Ulloa ${ }^{2}$, Richard R. Lane ${ }^{13}$, Dante Minnti ${ }^{13}$, Alexandre \\ Roman-Lopes $^{15}$, Andrés Almeida ${ }^{15}$, AND E. Moreno ${ }^{16}$ \\ ${ }^{1}$ School of Physics and Astronomy, Sun Yat-sen University, Zhuhai 519082, China \\ ${ }^{2}$ Departamento de Astronomía, Casilla 160-C, Universidad de Concepción, Concepción, Chile \\ ${ }^{3}$ Instituto de Astrofísica de Canarias, 38205 La Laguna, Tenerife, Spain \\ ${ }^{4}$ Departamento de Astrofísica, Universidad de La Laguna, 38206 La Laguna, Tenerife, Spain \\ ${ }^{5}$ ELTE Eötvös Loránd University, Gothard Astrophysical Observatory, Szombathely, Hungary \\ ${ }^{6}$ Space Telescope Science Institute, 3700 San Martin Drive, Baltimore, MD 21218, USA \\ ${ }^{7}$ Department of Physics and JINA Center for the Evolution of the Elements, University of Notre Dame, Notre Dame, IN 46556 USA \\ ${ }^{8}$ Astrophysics Research Institute, Liverpool John Moores University, Liverpool, United Kingdom \\ ${ }^{9}$ New Mexico State University, Las Cruces, NM 88003, USA \\ ${ }^{10}$ Institut Utinam, CNRS UMR 6213, Université Bourgogne-Franche-Comté, OSU THETA Franche-Comté, Observatoire de Besançon, \\ BP 1615, 25010 Besancon Cedex, France \\ ${ }^{11}$ Department of Astronomy, University of Texas at Austin, Austin, TX 78712, USA \\ ${ }^{12}$ Department of Astronomy, University of Virginia, Charlottesville, VA 22904-4325, USA \\ ${ }^{13}$ Instituto de Astrofisica, Pontificia Universidad Católica de Chile, Av. Vicuna Mackenna 4860, 782-0436 Macul, Santiago, Chile \\ ${ }^{14}$ Department of Physics and Astronomy, Universidad de La Serena, Cisternas 1200, La Serena, Chile \\ ${ }^{15}$ Instituto de Investigación Multidisciplinario en Ciencia y Tecnología, Universidad de La Serena, Benavente 980, La Serena, Chile \\ ${ }^{16}$ Instituto de Astronomía, Universidad Nacional Autónoma de México, Apartado Postal 70-264, 04510-México DF, Mexico and \\ ${ }^{17}$ Premium Postdoctoral Fellow of the Hungarian Academy of Sciences \\ Draft version October 10, 2018
}

\begin{abstract}
Metal-poor globular clusters (GCs) exhibit intriguing Al-Mg anti-correlations and possible Si-Al correlations, which are important clues to decipher the multiple-population phenomenon. NGC 5053 is one of the most metal-poor GCs in the nearby Universe, and has been suggested to be associated with the Sagittarius (Sgr) dwarf galaxy, due to its similarity in location and radial velocity with one of the Sgr arms. In this work, we simulate the orbit of NGC 5053, and argue against a physical connection between Sgr and NGC 5053. On the other hand, the Mg, Al, and Si spectral lines, which are difficult to detect in the optical spectra of NGC 5053 stars, have been detected in the near-infrared APOGEE spectra. We use three different sets of stellar parameters and codes to derive the $\mathrm{Mg}, \mathrm{Al}$, and $\mathrm{Si}$ abundances. Regardless of which method is adopted, we see a large Al variation, and a substantial Si spread. Along with NGC 5053, metal-poor GCs exhibit different Mg, Al, and Si variations. Moreover, NGC 5053 has the lowest cluster mass among the GCs that have been identified to exhibit an observable Si spread until now.
\end{abstract}

Keywords: globular clusters: individual: NGC 5053 - stars: abundances - stars: evolution

\section{INTRODUCTION}

The multiple-population (MP) phenomenon has now been observed in most of the evolutionary sequences of globular clusters (GCs): main-sequence (MS), subgiant branch (SGB), red giant branch (RGB), horizontal branch (HB), and asymptotic giant branch (AGB). While photometry is an efficient way of revealing MPs in most stellar phases, and particularly, fainter phases (e.g., Milone et al. 2015, Piotto et al. 2015), high-resolution spectroscopy allows deeper insights into GC formation and internal stellar evolution, providing detailed abundances for a number of elements with a variety of nucleosynthetic origins (e.g., García-Hernández et al. 2015, Mészáros et al. 2015; Schiavon et al. 2017; Tang et al. 2017). The $\mathrm{Al}-\mathrm{Mg}$ anti-correlations, and the less frequent $\mathrm{Si}-\mathrm{Al}$ correlations are of great interest, because these chemical patterns indicate that the polluting stars must have reached temperatures above 90 MK (Prantzos et al. 2007, Ventura et al. 2012, D'Antona et al. 2016), which is a strong constraint on the nature of these stars.
A significant silicon spread is observed in a few GCs, mainly metal-poor or massive GCs (e.g., Carretta et al. 2009b; Mészáros et al. 2015). Further investigations of metal-poor GCs would tell us more about the environment that stimulates the $\mathrm{Si}$ variation.

NGC 5053 is one of the most metal-poor GCs known $([\mathrm{Fe} / \mathrm{H}]=-2.27$, Harris 1996, 2010 version). It is located close to the Galactic north pole $\left(\alpha_{\mathrm{J} 2000}=13^{\mathrm{h}} 16^{\mathrm{m}} 27.1^{\mathrm{s}}\right.$, $\left.\delta_{\mathrm{J} 2000}=+17^{\circ} 42^{\prime} 01^{\prime \prime}, l=335.70^{\circ}, b=+78.95^{\circ}\right)$. With similar location and radial velocity ${ }^{1}$ (RV) to one of the Sagittarius (Sgr) arms (Law \& Majewski 2010), NGC 5053 has been speculated to be associated with the Sgr stream, but a firm association is still under debate (Palma et al. 2002: Bellazzini et al. 2003, Law \& Majewski 2010). A similar discussion is also found for its close companion, M53 (Figure 1). NGC 5053 and M53 have similar Galactocentric radius (17.8 and $18.4 \mathrm{kpc}$, respectively). Based on the $\mathrm{X}, \mathrm{Y}, \mathrm{Z}$ positions from the Har-

${ }^{1}$ The radial velocities in this work are heliocentric radial velocities. 
ris catalog, these two GCs are only $\sim 500$ pc away from each other currently. Furthermore, Chun et al. (2010) suggested that these two GCs also share a tidal bridge. Palma et al. (2002) suggested the Sgr association is unlikely for these two GCs because they are more metal poor than previously identified clusters $([\mathrm{Fe} / \mathrm{H}]=-2.27$ for NGC 5053 and $[\mathrm{Fe} / \mathrm{H}]=-2.10$ for M53, Harris cata$\log$ ). Bellazzini et al. (2003) considered NGC 5053 as one of the Sgr GCs based on its location and radial velocity, while M53 was not discussed. Law \& Majewski (2010) showed that the proper motion of M53 along the right ascension axis $\left(\mu_{\alpha} \cos \delta\right)$ is not consistent with any of the Sgr tidal arms, and concluded that M53 is probably not physically associated with Sgr. Meanwhile, the location and radial velocity of NGC 5053 are consistent with the T1 Sgr tidal arm, but the cluster's proper motion was not available at that time. Below, we simulate the orbit of NGC 5053 using newly obtained proper motion ${ }^{2}$ and our new software for dynamical modeling GravPot 16 (Fernández-Trincado et al., in prep.).

In terms of chemical abundances, Sbordone et al. (2015) analyzed HIRES spectra $(438-678 \mathrm{~nm}, R=$ 48000 ) downloaded from the Keck Observatory archive for one RGB star in NGC 5053. Because the spectral lines are generally weak in metal-poor stars, only 13 element species were investigated. We note that $\mathrm{Mg}, \mathrm{Al}$, and $\mathrm{Si}$ are not in their list of elements. Boberg et al. (2015, B15) observed 17 member stars using the Wisconsin-Indiana-Yale-NOAO-Hydra (WIYN) multi-object spectrograph. After analyzing the mediumresolution spectra $(R \sim 13000)$, they measured the abundances of $\mathrm{Fe}, \mathrm{Ca}, \mathrm{Ti}, \mathrm{Ni}, \mathrm{Ba}, \mathrm{Na}$, and $\mathrm{O}$. Boberg et al. found bimodality in the Na distribution, suggesting the existence of multiple populations in this GC. Previous work on elemental abundances provides evidence for a possible chemical connection between NGC 5053 and Sgr, including a very low yttrium abundance (Sbordone et al. 2015). However, a firm association has not been obtained yet. In this work, we focus on deriving the $\mathrm{Mg}, \mathrm{Al}$, and $\mathrm{Si}$ abundances from the APOGEE (Apache Point Observatory Galactic Evolution Experiment) spectra (Section 5), where relatively strong spectral lines are seen for these elements. These are the first measurements of $\mathrm{Mg}, \mathrm{Al}$, and $\mathrm{Si}$ in NGC 5053. These abundances are important indicators of the nature of the polluting stars that are responsible for multiple populations (Section 6).

\section{SAMPLE SELECTION AND DATA REDUCTION}

APOGEE (Majewski et al. 2017) was one of the programs operating during the Sloan Digital Sky Survey III (SDSS-III, Eisenstein et al. 2011). The multi-object NIR fiber spectrograph on the $2.5 \mathrm{~m}$ telescope at Apache Point Observatory (Gunn et al. 2006) delivers highresolution $(R \sim 22,500) H$-band spectra $(\lambda=1.51-1.69$ $\mu \mathrm{m})$, and the APOGEE survey targeted a color-selected sample that predominantly consists of RGB stars across the Milky Way (Zasowski et al. 2017). APOGEE data reduction software is applied to reduce multiple $3 \mathrm{D}$ raw data cubes into calibrated, well-sampled, combined 1D spectra (Nidever et al. 2015). In addition, the APOGEE Stellar Parameter and Chemical Abundances Pipeline (ASPCAP; García Pérez et al. 2016) derives

2 The proper motions in this work are absolute proper motions.

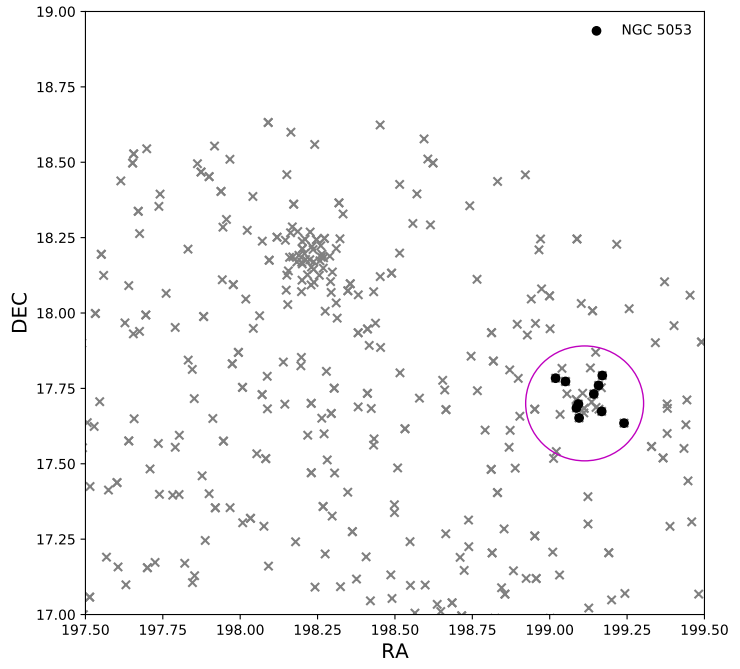

Figure 1. Locations of the stars in the APOGEE field of NGC 5053. The NGC 5053 member stars are indicated by filled circles. The magenta circle indicates the cluster tidal radius. Note that the star cluster in the upper left is M53.

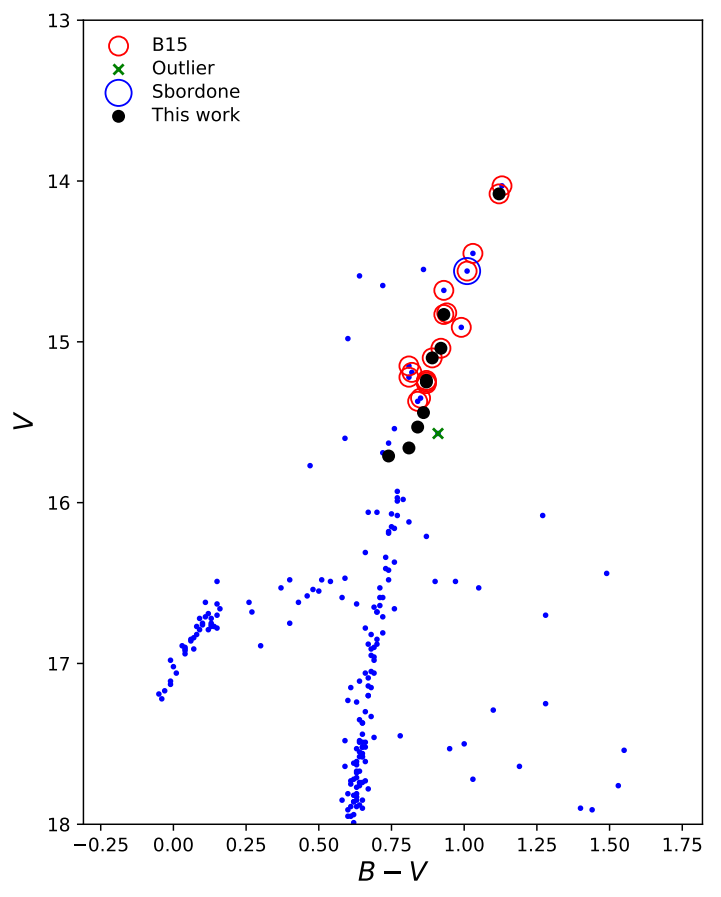

Figure 2. V versus B-V CMD of NGC 5053. The APOGEE selected members are indicated by black dots. The red open circles show the stars observed by B15. The blue open circle is the one RGB star observed in Sbordone et al. (2015). The cross is the one star that we exclude by CIMD and isochrone selection. 
Table 1

Photometric Properties of the NGC 5053 Members.

\begin{tabular}{|c|c|c|c|c|c|c|c|c|c|}
\hline \# & APOGEE_ID & $\mathrm{RA}_{\mathrm{J} 2000}\left(^{\circ}\right)$ & $\mathrm{DEC}_{\mathrm{J} 2000}\left({ }^{\circ}\right)$ & $B$ & $V$ & 2MASS $J$ & 2MASS $H$ & 2 MASS $K_{S}$ & B15 ID \\
\hline Star1 & $2 \mathrm{M} 13160457+1747017$ & 199.019064 & 17.783821 & 16.37 & 15.53 & 13.775 & 13.208 & 13.116 & \\
\hline Star2 & $2 \mathrm{M} 13161223+1746228$ & 199.050959 & 17.773018 & 15.20 & 14.08 & 11.956 & 11.377 & 11.243 & 3 \\
\hline Star3 & $2 \mathrm{M} 13162073+1741059$ & 199.086404 & 17.684973 & 15.99 & 15.10 & 13.293 & 12.752 & 12.633 & 15 \\
\hline Star4 & $2 \mathrm{M} 13162226+1741536$ & 199.092771 & 17.698231 & 16.12 & 15.25 & 13.140 & 12.776 & 12.608 & 20 \\
\hline Star5 & $2 \mathrm{M} 13162279+1739074$ & 199.094981 & 17.652067 & 16.30 & 15.44 & 14.101 & 13.655 & 13.513 & \\
\hline Star6 & $2 \mathrm{M} 13163419+1743530$ & 199.142482 & 17.731409 & 16.45 & 15.71 & 13.471 & 12.873 & 12.856 & 19 \\
\hline Star7 & $2 \mathrm{M} 13163790+1745355$ & 199.157922 & 17.759867 & 16.11 & 15.24 & 13.180 & 12.637 & 12.541 & 14 \\
\hline Star8 & $2 \mathrm{M} 13164030+1740254$ & 199.167950 & 17.673727 & 15.96 & 15.04 & 13.926 & 13.424 & 13.348 & $\ldots$ \\
\hline Star9 & $2 \mathrm{M} 13164077+1747338$ & 199.169899 & 17.792746 & 16.47 & 15.66 & 13.345 & 12.793 & 12.688 & $\ldots$ \\
\hline Star10 & $2 \mathrm{M} 13165764+1738050$ & 199.240180 & 17.634743 & 15.76 & 14.83 & 12.962 & 12.388 & 12.295 & 11 \\
\hline
\end{tabular}

Note: $B$ and $V$ apparent magnitudes come from Sarajedini \& Milone (1995).

stellar parameters and elemental abundances by comparing observed spectra to libraries of theoretical spectra (Zamora et al. 2015), constructed using extensive molecular/atomic linelists (Shetrone et al. 2015), in order to find the closest model match, using $\chi^{2}$ minimization in a multidimensional parameter space. In the current public SDSS Data Release 14 (DR14), up to more than 20 chemical elements were identified, and measured abundances provided (Holtzman et al. 2015; in prep.). DR14 includes all APOGEE-1 data, and APOGEE-2N data taken between July 2014 and July 2016.

To select stars that are members of NGC 5053, we first restrict the sample to stars within the tidal radius (11.43') given by Harris (1996; 2010 version). Then we exclude probable field stars by applying two generous criteria: $[\mathrm{Fe} / \mathrm{H}]_{\mathrm{NGC5053}} \pm 0.3, \mathrm{RV}_{\mathrm{NGC5053}} \pm 15 \mathrm{~km} / \mathrm{s}$, where $[\mathrm{Fe} / \mathrm{H}]_{\mathrm{NGC5053}}=-2.27$ and $\mathrm{RV}_{\mathrm{NGC5053}}=44.0$ $\mathrm{km} / \mathrm{s}$ from the Harris catalog. We identify 11 stars using the metallicities and RVs provided by APOGEE DR14. Given that NGC 5053 may have tidal structure (Lauchner et al. 2006), we also relax the search area to 3 times the tidal radius, but no further members in DR14 are identified in this way. Since NGC 5053 is far away from the Galactic plane, relaxing the $[\mathrm{Fe} / \mathrm{H}]$ and $\mathrm{RV}$ criteria $( \pm 0.6$ dex and $\pm 30 \mathrm{~km} / \mathrm{s})$ also do not increase the number of members. After checking the color-magnitude diagram (CMD) and stellar parameters, we further exclude one star (APOGEE_ID=2M13161337+1743552), because its location in the CMD is away from the RGB (Figure 2), and its ASPCAP stellar parameters also deviate from the isochrone (Figure 6). The stellar parameters are given in Section 4. Thus, our final list consists of 10 stars (Figure 1). We have also checked the photometry from P. Stetson ${ }^{3}$ for possible AGB (Asymtoptic Giant Branch) stars in our sample. García-Hernández et al. (2015) showed that $U$ vs. $(U-I)$ CMD is able to distinguish AGB stars from RGB stars. We have checked the $U$ vs. $(U-I)$ CMD for NGC 5053, where eight stars of our sample are identified. No obvious AGB star is found. Among our ten sample stars, six of them were also selected as members in B15 (Table1, also see Figure 2 .

\section{TRACKING THE ORBIT OF NGC 5053} cnrc.gc.ca/en/community/STETSON/standards /

\subsection{Adopted observational data for NGC 5053 and Sagittarius}

To obtain robust estimates of the orbit of NGC 5053, we employed recent high-quality data. First, we adopted the new proper motion measurements based on multiepoch Hubble Space Telescope (HST) observations separated by $\sim 11$ years in time. Details of this measurement, including the proper motion result for NGC 5053, will be presented in a forthcoming paper (Sohn et al., in prep). In short, we utilized the same established method of Sohn et al. (2012, 2013, 2017) to achieve a 1-d proper motion uncertainty of $\sim 0.07$ mas $y^{-1}$ for NGC 5053. The only other proper motion measurement found in the literature is provided by Kharchenko et al. (2013), and for comparison, we integrate orbits using their measurement separately (see Figure 3 for details).

The heliocentric distance of NGC 5053 was presented in Harris catalog and Arellano Ferro et al. (2010) using RR Lyrae stars. Being standard candles, RR Lyrae stars are particularly suitable to derive cluster distances with a high degree of confidence. Harris catalog suggested a distance of $16.4 \mathrm{kpc}$ for NGC 5053, which assumes $V(\mathrm{HB})=$ 16.65 (Sarajedini \& Milone 1995), $[\mathrm{Fe} / \mathrm{H}]=-2.29$, extinction ratio $R=3.0, E^{\prime}(B-V)=0.04$, and the relation $M_{V}=0.15[\mathrm{Fe} / \mathrm{H}]+0.80$. Arellano Ferro et al. (2010) derived a mean distance modulus of $16.12 \pm 0.4$, which correspond to a distance of $16.7 \pm 0.3 \mathrm{kpc}$. They assumes $[\mathrm{Fe} / \mathrm{H}]=-1.97, R=3.2, E(B-V)=0.018$. Even though different parameters were assumed in two literature studies, the derived distances agree with each other. We adopt the latter distance $16.7 \pm 0.3 \mathrm{kpc}$ in this work.

Using the APOGEE spectra, we have visually inspected the strong $\mathrm{Mg}, \mathrm{Al}$, and $\mathrm{Si}$ spectral lines (section 5), and list the derived RV in Table 3 . We obtain a mean RV of $43.4 \pm 2.2 \mathrm{~km} / \mathrm{s}$, which is consistent with the value from the Harris catalog $(44.0 \pm 0.4 \mathrm{~km} / \mathrm{s})$. The detailed values that we adopted in our simulations are shown in Table 2 .

\subsection{Orbits and Implications}

By combining our Milky Way potential model (see Appendix) with measurements of radial velocity, absolute proper motion, distance and sky position for NGC 5053 and Sagittarius, we ran $N_{\text {total }}=1 \times 10^{4}$ pairs of orbits NGC 5053 - Sagittarius, taking into account the uncertainties in the input data considering $1 \sigma$ variations in a Gaussian Monte Carlo approach. For each generated set 

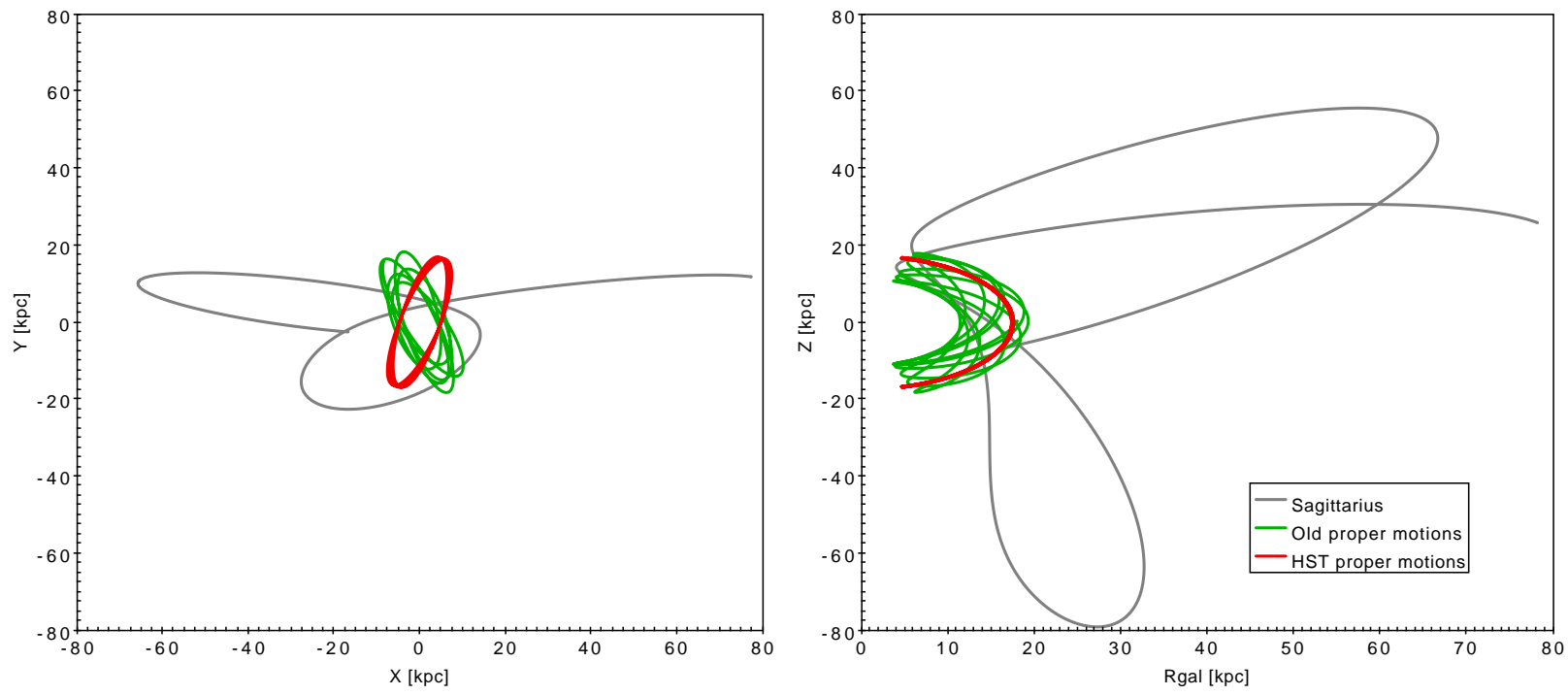

Figure 3. Representative orbits of Sgr (grey), NGC 5053 with HST proper motion (red), and NGC 5053 with old proper motion (green) in the reference frame where the bar is at rest. Left: Equatorial Galactic orbits. Right: Meridional Galactic orbits. The orbits of NGC 5053 do not resemble that of Sgr, suggesting the proposed connection between these two systems may be unlikely.

Table 2

RVs, Distances, and Proper Motions Adopted in This Work.

\begin{tabular}{ccccccccc}
\hline \hline$\#$ & RV & RV Err & d & d Err & $\mu_{\alpha} \cos \delta$ & $\mu_{\alpha} \cos \delta$ Err & $\mu_{\delta}$ & $\mu_{\delta}$ Err \\
\hline & \multicolumn{2}{c}{$(\mathrm{km} / \mathrm{s})$} & \multicolumn{2}{c}{$(\mathrm{pc})$} & \multicolumn{3}{c}{$(\mathrm{mas} / \mathrm{yr})$} & $(\mathrm{mas} / \mathrm{yr})$ \\
\hline Sgr & 171.0 & 17.0 & 24.8 & 0.8 & -2.75 & 0.2 & -1.65 & 0.22 \\
NGC 5053 old proper motion & 43.4 & 2.2 & \multirow{2}{*}{16.7} & 0.3 & -5.81 & 0.53 & -2.76 & 0.53 \\
NGC 5053 HST proper motion & & & & \multicolumn{5}{c}{ Sohn et al. (in prep.) } \\
\hline
\end{tabular}

Note: For NGC 5053, RV comes from APOGEE observation; distance comes from Arellano Ferro et al. (2010); the old proper motion comes from Kharchenko et al. (2013); the HST proper motion will be shown in Sohn et al. (1n prep.)

For Sgr, RV comes trom Kunder \& Chaboyer (2009); distance comes from Law \& Majewski (2010); proper motion comes from Pryor et al. (2010).

of parameters the orbit of NGC 5053 was computed backward in time, up to $2.5 \mathrm{Gyr}$. All orbital computations were made with a Runge-Kutta algorithm of seventh order (Fehlberg et al. 1968), with a variable time step determined at the initial conditions and conserved to 1 part in $10^{-14}$.

From the integrated set of orbits, we compute (1) the orbital eccentricity, defined as $e=$ $\left(r_{\text {apo }}-r_{p e r}\right) /\left(r_{\text {apo }}+r_{p e r}\right)$, with $r_{\text {apo }}$ and $r_{p e r}$ as successive apogalactic and perigalactic distances, (2) the maximun vertical amplitude $Z_{\max }$ as well as (3) the orbital Jacobi constant per unit mass, $E_{J}$, and (4) the "characteristic" orbital energy, $\left(E_{\min }+E_{\max }\right) / 2$, as defined in Moreno et al. (2015).

The major assumptions and limitations in our computations are: $i$ ) we do not consider the effects of dynamical friction of NGC 5053 and Sagittarius, which is expected to bring both systems closer to the Galactic center; ii) we ignore secular changes in our Milky Way potential over time, which are expected although the Milky Way has had a quiet recent accretion history; iii) we ignore the possibility that the pair NGC 5053 - Sagittarius were likely more massive in the past and have tidally lost some of that mass, which reduces the absolute probabilities we find.

Our simulation results (Figure 3) show that the orbit of NGC 5053 does not resemble that of Sgr, suggesting no association between the two objects. Fig- ure 4 shows results for the "characteristic" orbital energy $\left(E_{\max }+E_{\min } / 2\right)$ versus the orbital Jacobi energy $\left(E_{J}\right)$ in the potential mentioned above (including $1 \times 10^{4}$ random orbital realisations), the Galactic non-inertial frame is employed to compute the orbits. This plot shows a noticeable difference between NGC 5053 and Sgr. The Sgr occupies a very distinct region in these spaces, with an over-density at $E_{J} \sim-1400 \times 10^{5} \mathrm{~km}^{2} \mathrm{~s}^{-2}$ and $E_{\max }+E_{\min } / 2 \sim-1000 \mathrm{~km}^{2} \mathrm{~s}^{-2}$, with most of the NGC 5053 orbits shifted toward a lower distribution both in "characteristic" orbital energy and $E_{J}$. Therefore an association of NGC 5053 with Sgr appears implausible.

In Figure 5 we compared the eccentricity distribution for Sgr and NGC 5053 considering the HST and Kharchenko et al. (2013) absolute proper motions. This figure reassuringly shows that the eccentricity distributions of NGC 5053 and Sgr are decidedly different, even after taking errors into account, which means that NGC 5053 is characterized by different orbital configurations than Sgr. Therefore, our results suggest that it is unlikely that NGC 5053 comes from a dwarf galaxy (Sgr) that, on average, has significantly higher orbital energy.

\section{CHEMICAL ABUNDANCE DERIVATION}

Since some of the spectral lines in metal-poor stars become too weak for analysis, stellar parameters $\left(\mathrm{T}_{\text {eff }}\right.$, $\log g,[\mathrm{Fe} / \mathrm{H}])$ derived from $\chi^{2}$ fitting in ASPCAP may be problematic. Mészáros et al. (2015) suggested that manual analysis is required for stars more metal-poor than 


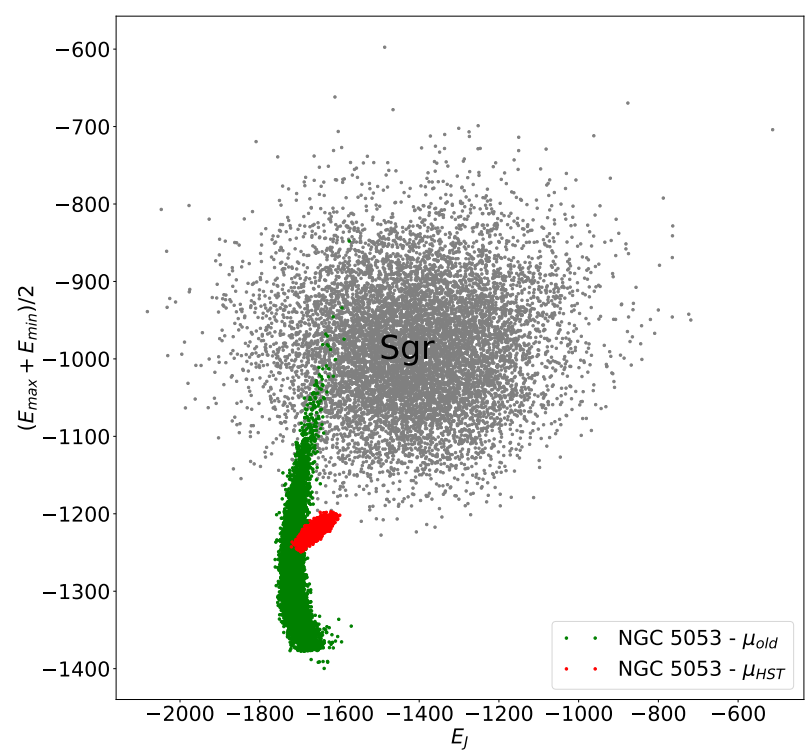

Figure 4. This diagram plots the "characteristic" orbital energy $\left(E_{\min }+E_{\max }\right) / 2$ versus the orbital Jacobi constant $\left(E_{J}\right)$, in units of $1 \times 10^{5} \mathrm{~km}^{2} \mathrm{~s}^{-2}$, in the reference frame of the bar, and considering $1 \sigma$ variations in a Gaussian Monte Carlo sampling $\left(1 \times 10^{4}\right.$ orbits). Grey dots are for the Sagittarius dwarf galaxy, green dots for NGC 5053 considering the absolute proper motion from Kharchenko et al. (2013), and red dots considering absolute proper motion from HST observations.

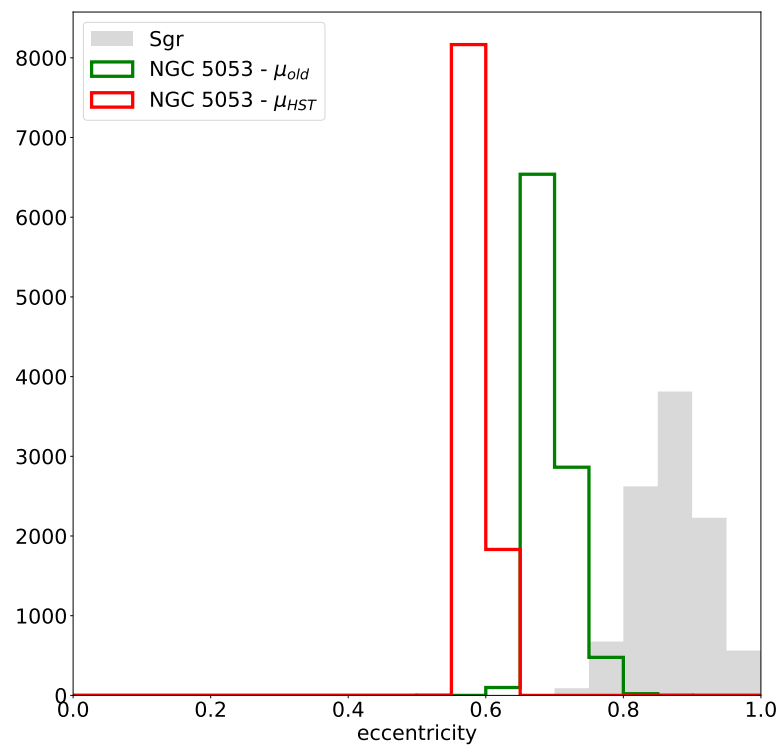

Figure 5. Histogram of the distribution of eccentricities for NGC 5053 and Sagittarius considering $1 \sigma$ variations in a Gaussian Monte Carlo sampling $\left(1 \times 10^{4}\right.$ orbits). The Sagittarius eccentricity is shown with grey histogram, while NGC 5053 adopting HST absolute proper motion are represented with a red histogram, and from adopted absolute proper motion from Kharchenko et al. (2013) is represented with a green histogram.

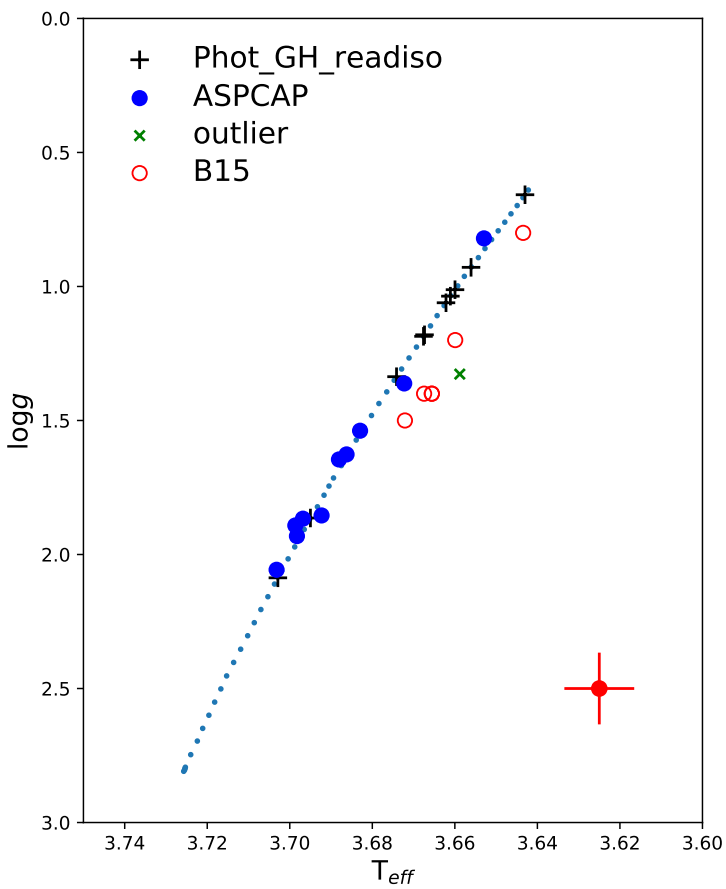

Figure 6. $\mathrm{T}_{\text {eff }}$ versus $\log g$. The ASPCAP results are labelled with blue dots. The error bars for ASPCAP results are shown in the bottom-right. The photometric stellar parameters using the González Hernández \& Bonifacio (2009) relations are labelled with plus signs. The B15-derived stellar parameters of the stars in common between our work and B15 are labelled with open circles. The DSED isochrone of $13 \mathrm{Gyr}$ and $[\mathrm{Fe} / \mathrm{H}]=-2.27,[\alpha / \mathrm{Fe}]=$ +0.20 is shown as a dotted line. The cross is the one star that we exclude by CMD and isochrone selection.

$[\mathrm{Fe} / \mathrm{H}]=-1$. Furthermore, some of the windows for a given element may be dominated by noise when the signal is too weak, which complicates the abundance derivation. Therefore, we derive chemical abundances manually for our sample of metal-poor stars in NGC 5053. However, note that all of our spectra have $\mathrm{S} / \mathrm{N}>75$ (Table 2), and most have $\mathrm{S} / \mathrm{N}>100$.

First, we estimated the stellar parameters using $B, V, I$ (Sarajedini \& Milone 1995) and 2MASS $J, H, K$ photometry (Table 1). The photometric $\mathrm{T}_{\text {eff }}$ is the mean value of the $\mathrm{T}_{\text {eff }}$ calculated with $B-V$ and $V-K_{s}$ colors using the equations of González Hernández \& Bonifacio (2009). The foreground extinction for each band is given by Schlafly \& Finkbeiner $(2011)^{4}$ and is very low $(E(B-V)=0.015)$. To derive $\log g$, we use the Dartmouth Stellar Evolution Database (DSED, Dotter et al. 2008) isochrone of age $=13 \mathrm{Gyr},[\mathrm{Fe} / \mathrm{H}]=-2.27$, $[\alpha / \mathrm{Fe}]=+0.20$. The $\log g$ for each star is read from the isochrone for its given photometric $T_{\text {eff }}$.

Figure 6 shows the isochrone, stellar parameters (SPs) from ASPCAP and from photometry. We find that (1) the ASPCAP derived SPs agree well with the isochrone; (2) the photometric derived SPs also agree well with the isochrone by definition; (3) the B15 SPs have higher $\log g$

${ }^{4}$ NED. https://ned.ipac.caltech.edu 
or lower $\mathrm{T}_{\text {eff }}$ than the isochrone; (4) The number distribution of stars along the isochrone for ASPCAP SPs agrees well with that of CMD in Figure 2, while photometric derived SPs suggest most stars to have low $\log g$ and low $\mathrm{T}_{\text {eff }}$.

The iron abundances of NGC 5053 stars have been determined by several optical investigations (e.g., B15, Sbordone et al. 2015), and the results from the literature are generally around -2.1 to -2.5 (see B15 for a list of literature results). The value given in the Harris catalog is $[\mathrm{Fe} / \mathrm{H}]=-2.27$. After visually inspecting the iron lines in the spectra, we find that the iron lines are hard to detect even with $\mathrm{S} / \mathrm{N} \sim 100$. Given that the derived chemical abundances are affected by the uncertainties of adopted stellar parameters, we decided to derive the elemental abundances using three different sets of stellar parameters:

1. SP1: Photometric derived $\mathrm{T}_{\mathrm{eff}}$ and $\log g$ from the isochrone, $[\mathrm{Fe} / \mathrm{H}]$ derived from the iron lines;

2. SP2: ASPCAP-calibrated $\mathrm{T}_{\text {eff }}$ and $\log g$, but assumed $[\mathrm{Fe} / \mathrm{H}]=-2.27$;

\section{SP3: B15 derived stellar parameters.}

The micro-turbulence velocity $\left(v_{t}\right)$ is calculated using the equation from Mészáros et al. (2015):

$$
v_{t}=2.24-0.3 \times \log g
$$

After visually inspecting the APOGEE spectra of the member stars, we find that only $\mathrm{Mg}, \mathrm{Al}$, and Si can be reliably derived for most of the stars, due to their extreme metal-poor nature. We use the MARCS/Turbospectrum stellar libraries from Zamora et al. (2015), where we interpolate the atmospheric model spectra of each star from these model grids. The line list used in this work is the latest internal DR14 atomic/molecular linelist (linelist.20170418). The wavelengths of the line centers of the $\mathrm{Mg}, \mathrm{Al}$, and $\mathrm{Si}$ atomic lines are taken from Souto et al. (2016). We first inspect the strong lines, and apply additional RV shifts on top of the APOGEE RV correction if necessary. Note that APOGEE pipeline determined RV may have a small offset from the true value in the case of weak spectral lines. The line-by-line analysis is done using two codes: (1) A simple python script written by us, based on the 1D LTE spectral synthesis code Turbospectrum (Plez 2012). We call this TBS for short; and (2) BACCHUS, which is also based on Turbospectrum. We use TBS for stellar parameters SP2 and SP3, and use BACCHUS for stellar parameters SP1.

We first briefly introduce TBS. Each spectrum is normalized locally to avoid possible offset in flux, then the $\chi^{2}$ minimum is searched between the observed and model spectra. An example is given in Figure 7. At this stage, visual inspection is required to weed out extremely weak lines and bad fits. Finally, the means of individual measurements are taken as the chemical abundances, and standard deviations as errors (Table 6). For BACCHUS, readers are referred to Masseron et al. (2016) for a detailed description. Here we only briefly describe the basic features in BACCHUS. First, a sigma-clipping is applied on the selected continuum points around the targeted line, then a linear fit is computed over the remaining points. Line broadening effects include not only the one caused by microturbulence, but also instrumental broadening. The code can detect significant bad fits, like a sudden drop in the spectrum due to bad pixels in the detectors. Observed spectra and model spectra are compared in four different ways to determine abundances: $\chi^{2}$ minimization, line intensity, equivalent width, and spectral synthesis. We use $\chi^{2}$ minimization in this work for consistence between the two codes. Similar to TBS, the means of individual measurements are taken as the chemical abundances, and standard deviations as errors. Between these two codes, BACCHUS is faster and also more sophisticated in handling the continuum fitting, line broadening, and other issues, while visual inspection in TBS ensures that our results are not overly influenced by the noise in the spectra. The measured chemical abundances are given with respect to the solar abundances from Asplund et al. (2005).

\section{RESULTS}

\subsection{Multiple Populations in NGC 5053}

We derive the iron abundances using BACCHUS, listed in Table 4. The mean iron abundance is -2.20 with a standard deviation of 0.02 dex. Our measurements basically agree with the iron abundance given by the Harris catalog $([\mathrm{Fe} / \mathrm{H}]=-2.27)$. The iron lines in NIR are generally weaker and less numerous compared to those in the optical; therefore an offset of $\sim 0.1$ dex between the iron abundances derived from the NIR and from the optical is acceptable.

Visual inspection suggests that for three stars (Star5, Star8, Star9) we have no reliable Al measurements because the lines are too weak. However, we managed to inspect these lines individually to estimate upper limits. We plot the measured abundances and upper limits for the three different sets of stellar parameters in Figures 8. To study the multiple populations in metalpoor GCs in context, we also plot three metal-poor GCs (M15, M92, M53) from Mészáros et al. (2015) that have $[\mathrm{Fe} / \mathrm{H}]<-2$. The first and second generations that were defined in Mészáros et al. (2015) are distinguished by red circles and blue triangles, respectively. Interestingly, M53 $([\mathrm{Fe} / \mathrm{H}]=-2.10)$, which is a close companion of NGC 5053 in the sky, was also included in that work. We label the M53 stars with filled symbols. A clear Al variation as large as 0.8 dex is found in NGC 5053, and we can easily separate two groups of stars with different $\mathrm{Al}$ abundances. This is true for all three sets of SPs. We note that six stars are in common between our sample and B15 (Table 1), and four of them have $[\mathrm{Na} / \mathrm{Fe}]$ measurements from optical spectra (Table 5). The only first-generation (FG) star (Star2) in common shows a low $[\mathrm{Na} / \mathrm{Fe}](<0)$, and three second-generation $(\mathrm{SG})$ stars in common show $[\mathrm{Na} / \mathrm{Fe}] \sim 0.9$. Thus, our stellar generation division by $\mathrm{Al}$ is supported by the $\mathrm{Na}$ abundances from B15. The two stellar generations of NGC 5053 also fit well into the two stellar generations defined by the other metal-poor GCs, though we note that three FG stars in this work (Star2, Star8, Star9) lie at the limit that separates the two stellar generations, although two of these stars only have $\mathrm{Al}$ upper limits from our data and therefore could actually lie closer to the FG star region. Meanwhile, $\mathrm{Mg}$ shows no strong variation between the two stellar populations. In all three sets of measure- 
Table 3

Stellar Parameters of the NGC 5053 Members from ASPCAP.

\begin{tabular}{cccccccccc}
\hline \hline$\#$ & $\mathrm{RV}$ & $\delta_{\mathrm{RV}}$ & {$[\mathrm{Fe} / \mathrm{H}]$} & $\delta_{[\mathrm{Fe} / \mathrm{H}]}$ & \multicolumn{1}{c}{$\mathrm{T}_{\text {eff }}$} & $\delta_{\mathrm{T}_{\text {eff }}}$ & $\log g$ & $\delta_{\log g}$ & SNR \\
\hline \multicolumn{7}{c}{$(\mathrm{km} / \mathrm{s})$} & & \multicolumn{6}{c}{$(\mathrm{K})$} & & & \\
\hline Star1 & 43.81 & 0.17 & -2.28 & 0.02 & 4923.7 & 99.3 & 1.85 & 0.13 & 108 \\
Star2 & 44.56 & 0.06 & -2.36 & 0.02 & 4497.3 & 67.7 & 0.82 & 0.11 & 187 \\
Star3 & 45.19 & 0.06 & -2.24 & 0.02 & 4855.6 & 96.3 & 1.63 & 0.13 & 105 \\
Star4 & 46.02 & 0.15 & -2.31 & 0.02 & 4819.1 & 94.2 & 1.54 & 0.13 & 105 \\
Star5 & 47.12 & 0.10 & -2.52 & 0.03 & 4991.9 & 112.0 & 1.93 & 0.15 & 76 \\
Star6 & 40.68 & 0.13 & -2.35 & 0.02 & 4975.2 & 95.3 & 1.87 & 0.12 & 138 \\
Star7 & 41.97 & 0.18 & -2.25 & 0.02 & 4996.4 & 104.3 & 1.89 & 0.14 & 112 \\
Star8 & 41.86 & 0.38 & -2.48 & 0.03 & 5048.9 & 112.2 & 2.06 & 0.15 & 91 \\
Star9 & 40.77 & 0.15 & -2.44 & 0.02 & 4701.9 & 88.6 & 1.36 & 0.14 & 104 \\
Star10 & 41.81 & 0.10 & -2.35 & 0.02 & 4876.2 & 88.0 & 1.65 & 0.12 & 153 \\
\hline
\end{tabular}

Note: The RVs have been corrected by visually inspection of the strong Mg, Al, and Si spectral lines. See text for more details.
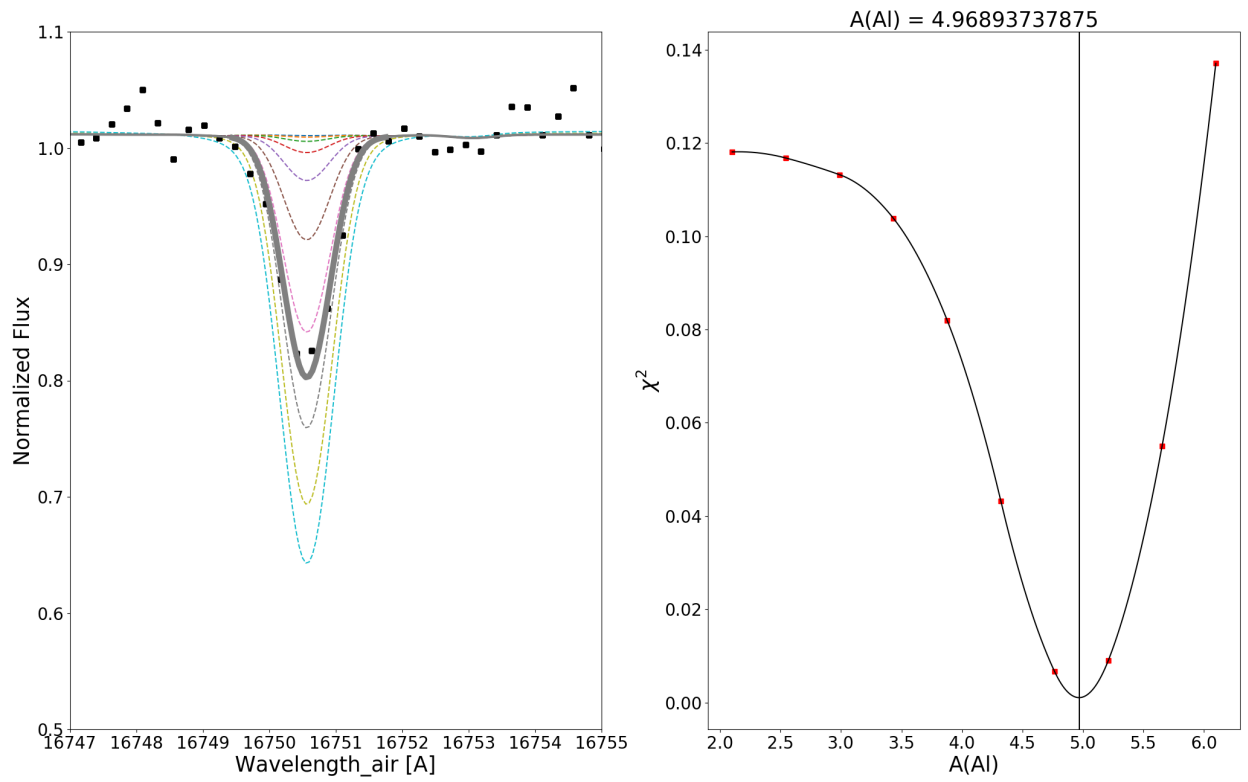

Figure 7. Example of spectral line fitting. In the left panel, the observed spectral line is shown as discrete dots, while models with different Al abundances are shown as lines with different colors. In the right panel, the $\chi^{2}$ of models with different Al abundances are plotted. A quadratic polynomical interpolation is used to find the $\chi^{2}$ minimum, as indicated by the vertical line.

Table 4

Stellar Parameters 1 (SP1).

\begin{tabular}{cccc}
\hline \hline$\#$ & $\mathrm{~T}_{\text {eff }}$ & $\log g$ & {$[\mathrm{Fe} / \mathrm{H}]$} \\
\hline star1 & 4722.05 & 1.34 & -2.18 \\
star2 & 4395.42 & 0.66 & -2.19 \\
star3 & 4648.89 & 1.18 & -2.21 \\
star4 & 4593.50 & 1.06 & -2.17 \\
star5 & 4954.46 & 1.86 & -2.20 \\
star6 & 4651.53 & 1.19 & -2.20 \\
star7 & 4570.49 & 1.01 & -2.19 \\
star8 & 5045.27 & 2.09 & -2.17 \\
star9 & 4529.76 & 0.93 & -2.24 \\
star10 & 4582.29 & 1.04 & -2.20 \\
\hline
\end{tabular}

Table 5

Stellar Parameters (SP3) and Abundances Derived from Optical Spectra (B15).

\begin{tabular}{ccccc}
\hline \hline$\#$ & $\mathrm{~T}_{\text {eff }}$ & $\log g$ & {$[\mathrm{Fe} / \mathrm{H}]$} & {$[\mathrm{Na} / \mathrm{Fe}]$} \\
\hline star2 & 4400 & 0.80 & -2.43 & $<0.0$ \\
star3 & 4630 & 1.40 & -2.48 & $\ldots$ \\
star4 & 4700 & 1.51 & -2.45 & 0.9 \\
star6 & 4650 & 1.40 & -2.45 & $\ldots$ \\
star7 & 4630 & 1.40 & -2.47 & 0.8 \\
star10 & 4570 & 1.20 & -2.44 & 1.0 \\
\hline
\end{tabular}

ments, we do not find stars with $[\mathrm{Mg} / \mathrm{Fe}]<0.0$. The $\mathrm{Mg}-\mathrm{Al}$ anti-correlation of NGC 5053 does not resemble that of M92 or M15 ([Fe/H] -2.3, open symbols), but 


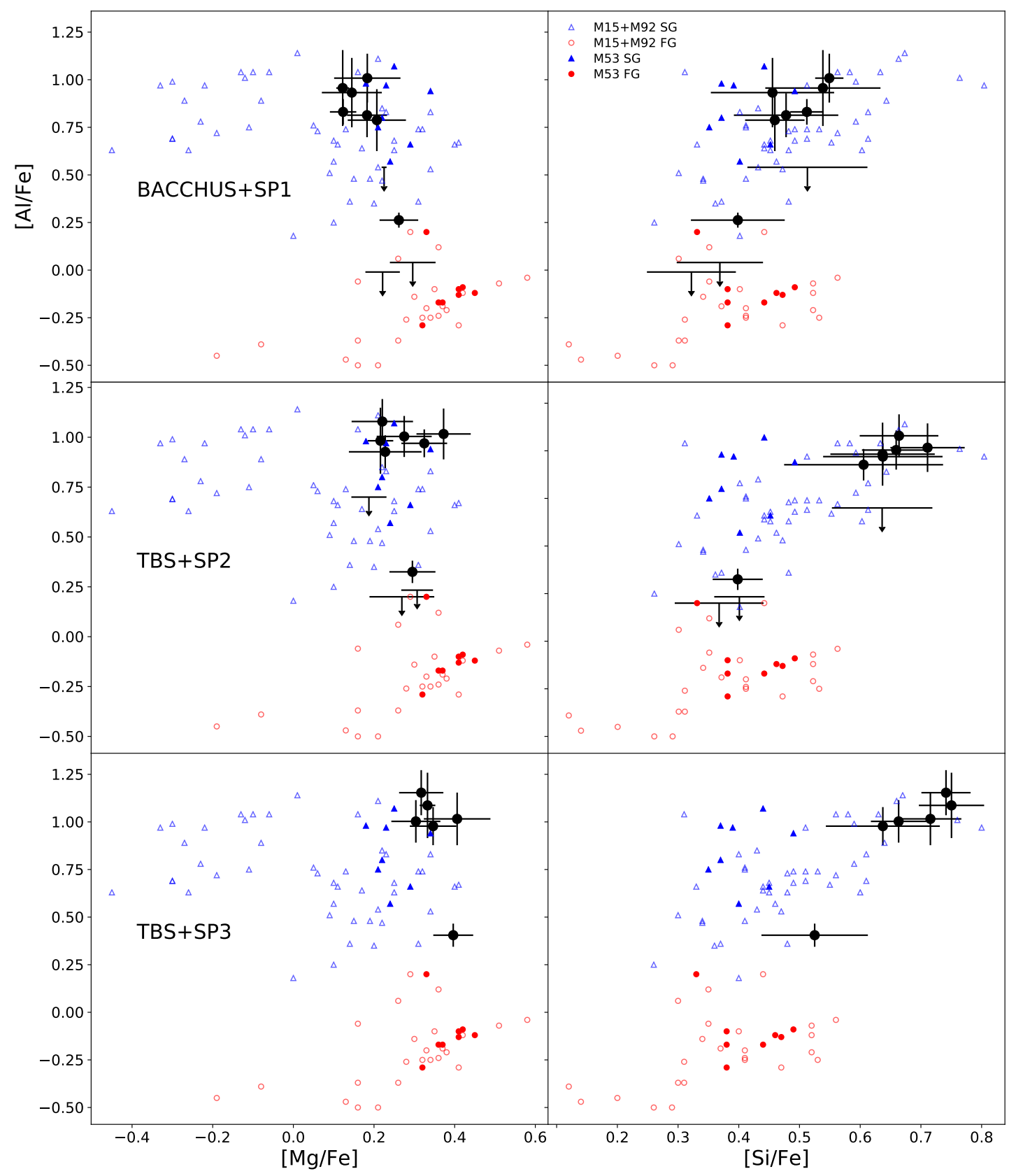

Figure 8. Left column: $[\mathrm{Al} / \mathrm{Fe}]$ versus $[\mathrm{Mg} / \mathrm{Fe}]$. Right column: $[\mathrm{Al} / \mathrm{Fe}]$ versue $[\mathrm{Si} / \mathrm{Fe}]$. Top row: SP1 and BACCHUS. Middle row: SP2 and TBS. Bottom row: SP3 and TBS. The NGC 5053 stars are black dots. The two generations of stars in the metal-poor GCs from Mészáros et al. (2015) are labelled as red circles (FG) and blue triangles (SG), with M15 and M92 as open symbols, and M53 as filled ones. Three stars with estimated $\mathrm{Al}$ upper limits are indicated by arrows. 
Table 6

Manually Derived Chemical Abundances of the NGC 5053

Members.

\begin{tabular}{|c|c|c|c|c|c|c|}
\hline \multicolumn{7}{|c|}{ BACCHUS+SP1 } \\
\hline \# & {$[\mathrm{Mg} / \mathrm{Fe}]$} & $\delta_{[\mathrm{Mg} / \mathrm{Fe}]}$ & {$[\mathrm{Al} / \mathrm{Fe}]$} & $\delta_{[\mathrm{Al} / \mathrm{Fe}]}$ & {$[\mathrm{Si} / \mathrm{Fe}]$} & $\delta_{[\mathrm{Si} / \mathrm{Fe}]}$ \\
\hline star1 & 0.14 & 0.07 & 0.93 & 0.18 & 0.45 & 0.10 \\
\hline star2 & 0.26 & 0.05 & 0.26 & 0.04 & 0.40 & 0.08 \\
\hline star3 & 0.18 & 0.08 & 1.01 & 0.13 & 0.55 & 0.02 \\
\hline star4 & 0.12 & 0.02 & 0.96 & 0.20 & 0.54 & 0.09 \\
\hline star5 & 0.22 & 0.01 & $\ldots$ & $\ldots$ & 0.51 & 0.10 \\
\hline star6 & 0.12 & 0.03 & 0.83 & 0.07 & 0.51 & 0.03 \\
\hline star7 & 0.21 & 0.07 & 0.79 & 0.16 & 0.46 & 0.05 \\
\hline star8 & 0.30 & 0.06 & $\ldots$ & $\ldots$ & 0.37 & 0.07 \\
\hline star9 & 0.22 & 0.04 & $\ldots$ & $\ldots$ & 0.32 & 0.07 \\
\hline star10 & 0.18 & 0.05 & 0.81 & 0.12 & 0.48 & 0.09 \\
\hline \multicolumn{7}{|c|}{ TBS+SP2 } \\
\hline \# & {$[\mathrm{Mg} / \mathrm{Fe}]$} & $\delta_{[\mathrm{Mg} / \mathrm{Fe}]}$ & {$[\mathrm{Al} / \mathrm{Fe}]$} & $\delta_{[\mathrm{Al} / \mathrm{Fe}]}$ & [Si/Fe] & $\delta_{[\mathrm{Si} / \mathrm{Fe}]}$ \\
\hline star1 & 0.23 & 0.09 & 0.93 & 0.08 & 0.60 & 0.13 \\
\hline star2 & 0.30 & 0.06 & 0.33 & 0.06 & 0.40 & 0.04 \\
\hline star3 & 0.22 & 0.08 & 1.08 & 0.11 & 0.66 & 0.06 \\
\hline star4 & 0.22 & 0.03 & 0.98 & 0.17 & 0.63 & 0.09 \\
\hline star5 & 0.19 & 0.04 & $\ldots$ & $\ldots$ & 0.63 & 0.08 \\
\hline star6 & 0.27 & 0.07 & 1.00 & 0.10 & 0.66 & 0.06 \\
\hline star7 & 0.37 & 0.07 & 1.02 & 0.13 & 0.71 & 0.06 \\
\hline star8 & 0.31 & 0.04 & $\ldots$ & $\ldots$ & 0.40 & 0.04 \\
\hline star9 & 0.27 & 0.08 & $\ldots$ & $\ldots$ & 0.37 & 0.07 \\
\hline star10 & 0.32 & 0.06 & 0.97 & 0.07 & 0.63 & 0.10 \\
\hline \multicolumn{7}{|c|}{ TBS+SP3 } \\
\hline$\#$ & {$[\mathrm{Mg} / \mathrm{Fe}]$} & $\delta_{[\mathrm{Mg} / \mathrm{Fe}]}$ & {$[\mathrm{Al} / \mathrm{Fe}]$} & $\delta_{[\mathrm{Al} / \mathrm{Fe}]}$ & [Si/Fe] & $\delta_{[\mathrm{Si} / \mathrm{Fe}]}$ \\
\hline star2 & 0.40 & 0.05 & 0.40 & 0.06 & 0.52 & 0.09 \\
\hline star3 & 0.32 & 0.05 & 1.15 & 0.12 & 0.74 & 0.04 \\
\hline star4 & 0.33 & 0.02 & 1.09 & 0.17 & 0.75 & 0.05 \\
\hline star6 & 0.30 & 0.06 & 1.00 & 0.11 & 0.66 & 0.05 \\
\hline star7 & 0.41 & 0.08 & 1.02 & 0.14 & 0.72 & 0.05 \\
\hline star10 & 0.35 & 0.06 & 0.98 & 0.10 & 0.64 & 0.09 \\
\hline
\end{tabular}

resembles that of M53 ([Fe/H] -2.1, filled symbols); in the sense that no strong Mg depletion is seen in NGC 5053, even in SG stars.

All three sets of chemical measurements suggest a substantial Si abundance variation $(\gtrsim 0.25 \mathrm{dex})$, which leads to a $\mathrm{Si}$-Al correlation. Note that the $\mathrm{Si}$ separation between the two stellar generations is smaller than the $\mathrm{Al}$ separation. In terms of Si distribution, NGC 5053 stars resemble those of M92 or M15, but not of M53. The different behaviors of $\mathrm{Mg}$ and $\mathrm{Si}$ among metal-poor GCs suggest that metallicity is not the only parameter that regulates the MP phenomenon (see Section 6.2).

\subsection{Robustness of the Measurements}

In this work, we derive elemental abundances adopting different sets of stellar parameters and different codes. SP3 (B15) usually yields higher elemental abundances due to the assumed low metallicities $([\mathrm{Fe} / \mathrm{H}] \sim-2.45)$. Conversely, SP1 usually yields lower elemental abundances: for example, the $[\mathrm{Mg} / \mathrm{Fe}]$ abundances are lower in the SG stars, where an Al-Mg anti-correlation is barely seen. Bearing these uncertainties in mind, our conclusions, namely, that there is a range of $\mathrm{Al}$ and $\mathrm{Si}$ abundances in NGC 5053 stars, and that those are correlated, are insensitive to the choice of stellar parameters adopted in the analysis.

Figure 9 compares the elemental abundances derived by ASPCAP and the BACCHUS+SP1 method. ASPCAP derived $[\mathrm{Mg} / \mathrm{Fe}]$ and $[\mathrm{Si} / \mathrm{Fe}]$ are higher than those of
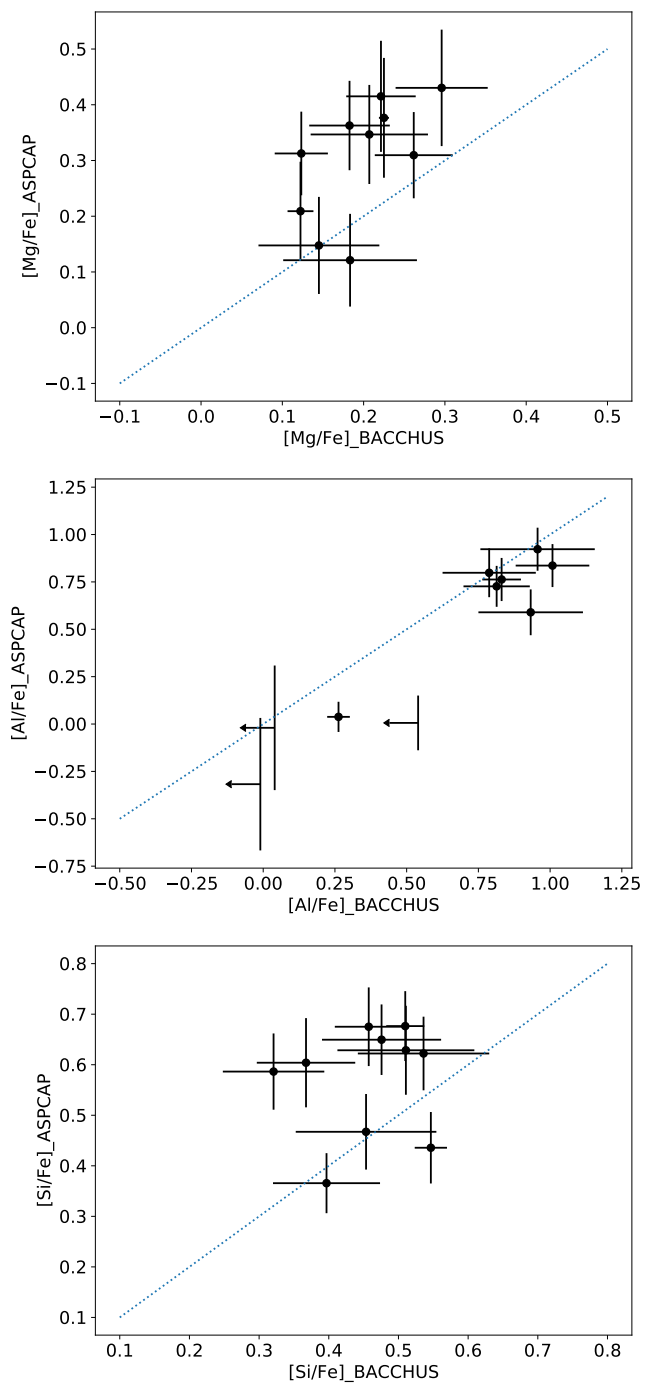

Figure 9. Comparison between the BACCHUS+SP1 and ASPCAP pipeline results. The dotted lines represent the one-to-one line.

the BACCHUS results, partially due to the lower $[\mathrm{Fe} / \mathrm{H}]$ from the ASPCAP pipeline $([\mathrm{Fe} / \mathrm{H}]=-2.36 \pm 0.09)$. There is no one-to-one correlation between the two sets of results. On the other hand, the $[\mathrm{Al} / \mathrm{Fe}]$ abundances are similar between the two methods, though we note that the upper limits of the chemical abundances can only be given after visual inspection under the circumstance of very weak lines. We find a similar situation if we compare the elemental abundances derived by ASPCAP and the TBS+SP2 method (Figure 10). Though the systematic offset between the $\mathrm{Mg}$ (and $\mathrm{Si}$ ) abundances from these two methods is smaller, their comparison does not show a one-to-one correlation. However, the $[\mathrm{Al} / \mathrm{Fe}]$ abundances 

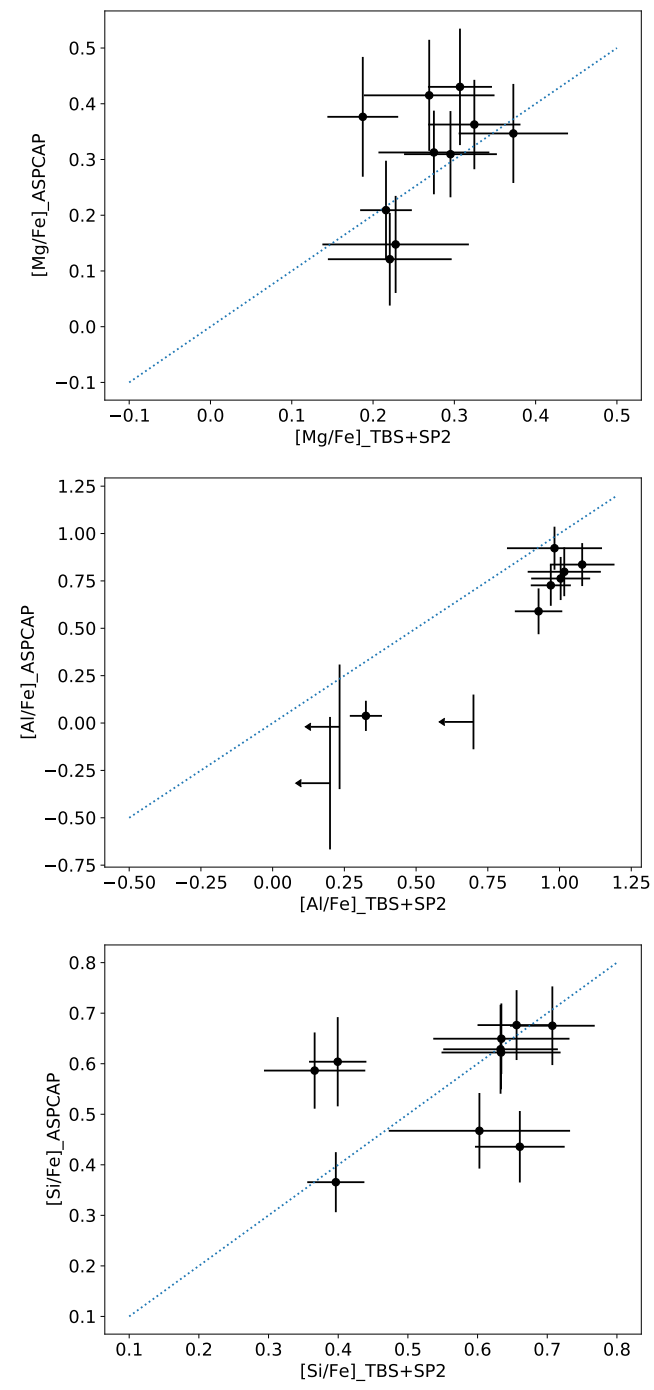

Figure 10. Comparison between the TBS+SP2 and ASPCAP pipeline results. The dotted lines represent the one-to-one line.

derived from the two methods follow a one-to one correlation, with possible systematic offset. Therefore, we emphasize that it is necessary to derive chemical abundances manually in the case of very metal-poor stars, especially for those stars with $[\mathrm{Fe} / \mathrm{H}]<-2$. We note that the ASPCAP performance at extremely low $[\mathrm{Fe} / \mathrm{H}]$ has been a known issue in the ASPCAP team.

\section{DISCUSSION}

\subsection{Is NGC 5053 Associated with Sgr?}

Using the newly obtained, high-precision proper motion of NGC 5053 and our software GravPot16, we have shown that the orbit of NGC 5053 is significantly dif- ferent from that of Sgr. Our simulation results strongly suggest against a connection between NGC 5053 and Sgr.

In terms of chemistry, we first take a look at $[\mathrm{Mg} / \mathrm{Fe}]$ vs. $[\mathrm{Fe} / \mathrm{H}]$, since the $\mathrm{Mg}$ abundances of NGC 5053 are newly obtained in this work, and Hasselquist et al. (2017) have shown that the Milky Way (MW) thin disk, thick disk, and Sgr core stars are distinguishable in this parameter space. To visualize the MW thin disk, thick disk and halo, we first select giants from APOGEE DR14 with the following criteria: (1) $3500<\mathrm{T}_{\text {eff }}<5000 \mathrm{~K}$; (2) $\log g<2.0$; (3) SNR $>70$; (4) $[\mathrm{Fe} / \mathrm{H}]$ and $[\mathrm{Mg} / \mathrm{Fe}]$ measurements are available. The number density of these stars on a logarithmic scale is indicated by a grey scale map in Figure 11, where high number density regions are darker. The stars selected by Hasselquist et al. are plotted as magenta triangles. Besides NGC 5053, we also try to identify other possible Sgr-associated GCs in APOGEE. We preliminarily identify five stars from M54, using a similar method to that described in Section 2. We have checked the strong $\mathrm{Mg}$ lines manually, and find that the ASPCAP fits are reasonable. Therefore, we use the ASPCAP values instead of performing another manual analysis. We note that more observations of M54 stars have been scheduled by the APOGEE team; a careful analysis of M54 stars will be left to a future publication. We also gather data for other possible Sgr-associated GCs from literature: Terzan 8 (Carretta et al. 2014), M54 (Carretta et al. 2010b), Palomar 12 (Cohen 2004), Terzan 7 (Sbordone et al. 2007), Arp 2 (Mottini et al. 2008), and NGC 6534 (Carretta et al. 2017). The literature $[\mathrm{Fe} / \mathrm{H}]$ and $[\mathrm{Mg} / \mathrm{Fe}]$ are shown as green squares in Figure 11 with associated measurement errors. Note that $[\mathrm{Fe} / \mathrm{H}]$ and $[\mathrm{Mg} / \mathrm{Fe}]$ measured by Carretta et al. (2010b) and APOGEE are consistent within their uncertainty ranges.

We see that the MW thin disk, thick disk, and the Sgr core sequences are indeed well separated at $[\mathrm{Fe} / \mathrm{H}]>$ -1.0 (Figure 11). At lower metallicity, MW thin disk and thick disk do not have much stars in this region. The stars between $-2.0<[\mathrm{Fe} / \mathrm{H}]<-0.9$ are suggested to be halo stars with two different chemical and dynamical properties (Hayes et al. 2018, Fernández-Alvar et al. 2018). We run out of Sgr stars at $[\mathrm{Fe} / \mathrm{H}]<-1.7$, and the MW halo sequence becomes wide and sparse, so it is difficult to estimate whether NGC 5053 is chemically similar to Sgr or MW halo using only $[\mathrm{Mg} / \mathrm{Fe}]$ and $[\mathrm{Fe} / \mathrm{H}]$. On the other hand, the possible Sgr-associated GCs loosely form a sequence in the low metallicity regime (green squares). The average $\mathrm{Mg}$ abundance of NGC 5053 appears lower than the barely-seen MW halo sequence and its value $(\sim 0.2 \mathrm{dex})$ is lower than that of Terzan $8([\mathrm{Mg} / \mathrm{Fe}]=0.47 \mathrm{dex})$, which has similar metallicity as NGC $5053([\mathrm{Fe} / \mathrm{H}]=-2.27$, Carretta et al. 2014). This indicates that NGC 5053 may be chemically different than other Sgr GCs, though we caution that the Mg abundances of SG stars may be slightly affected by the $\mathrm{Mg}-\mathrm{Al}$ reaction chains and that the Sgr GC trend is highly dependent on the three most metal-poor GCs. Meanwhile, studies of metals (e.g., Ni), and heavy elements (e.g., Y and Ba) in NGC 5053, have not shown conclusive evidence of a Sgr connection (Sbordone et al. 2015). Unfortunately, $\mathrm{Ni}$ and s-process elements (e.g., $\mathrm{Nd}$ and $\mathrm{Ce}$ ) do not show any measurable lines in our APOGEE spectra. 


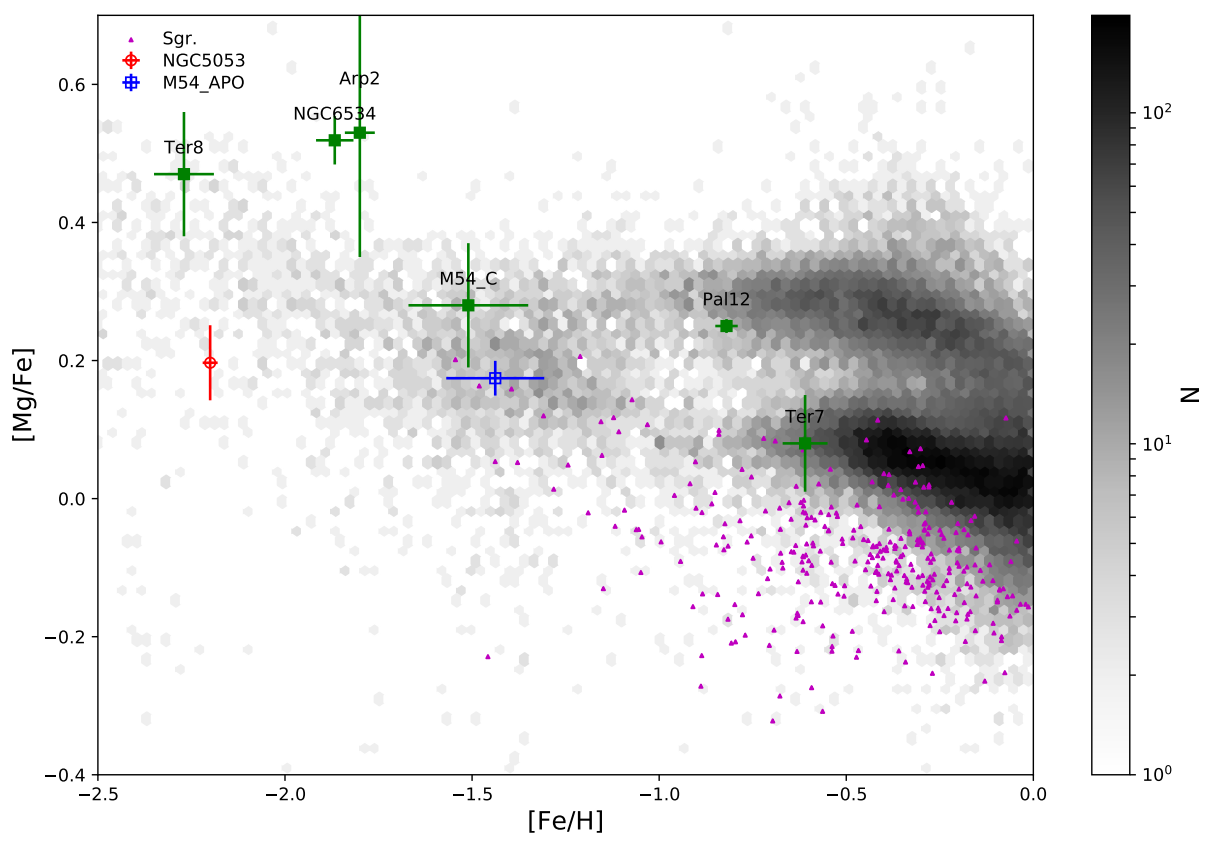

Figure 11. $[\mathrm{Mg} / \mathrm{Fe}]$ versus $[\mathrm{Fe} / \mathrm{H}]$. The Sgr stars selected by Hasselquist et al. (2017) are plotted as magenta triangles. The red open circle represents the average $[\mathrm{Mg} / \mathrm{Fe}]$ and $[\mathrm{Fe} / \mathrm{H}]$ abundances for $\mathrm{NGC} 5053$ (BACCHUS+SP1). The blue open square represents the average $[\mathrm{Mg} / \mathrm{Fe}]$ and $[\mathrm{Fe} / \mathrm{H}]$ abundances of the M54 members. GCs with possible Sgr origin are shown as green filled squares, and their GC names are labelled. We select a sample of stars from the APOGEE survey to visualize the thin disk, thick disk, and halo (see text). The grey scale map indicates the number density of these selected stars on a logarithmic scale.

To summarize, we find tentative chemical evidence to dismiss a connection between NGC 5053 and Sgr, and our simulated orbit of NGC 5053 also argues against such a connection.

\subsection{Multiple Populations in the Metal-Poor Regime}

After carefully inspecting three elements involved in the $\mathrm{Mg}-\mathrm{Al}$ nuclear chain for four metal-poor GCs with different GC cluster masses (Table 7), we see interesting behaviors in their abundance variations. The $\mathrm{Mg}-\mathrm{Al} \mathrm{nu}-$ clear chain and the associated Si leakage are supposed to be activated at temperature above 90 MK (Prantzos et al. 2007; Ventura et al. 2012; D'Antona et al. 2016), with stronger Si leakage at higher temperature. Strong $\mathrm{Mg}$ depletion is observed in the two more metalpoor $([\mathrm{Fe} / \mathrm{H}] \sim-2.3)$ and massive GCs M92 and M15, but not in the similar metallicity but less massive GC NGC 5053, or in the less metal-poor $([\mathrm{Fe} / \mathrm{H}] \sim-2.1)$ but massive GC M53. On the other hand, a Si spread is found for the three most metal-poor GCs M92, M15, and NGC 5053, regardless of their cluster masses, while a Si spread is not found in the less metal-poor, massive GC M53. Therefore, the correlations among Mg, Al, and Si for MPs are not only affected by metallicity, other parameters may also play important roles. Cluster mass has been suggested to be another parameter that affects the elemental abundances of MPs (e.g., Carretta et al. 2010a Pancino et al. 2017). In our work, we notice that NGC 5053 has a lower cluster mass (Table 7) and exhibits less prominent $\mathrm{Al}-\mathrm{Mg}$ anti-correlation compared to M15 and M92. This qualitatively agrees with the trend given in Pancino et al. (2017), where the standard deviation of the $|\mathrm{AI} / \mathrm{Mg}|$ distribution and its maximum variation of low mass GCs tend to be smaller in a given $[\mathrm{Fe} / \mathrm{H}]$.

Carretta et al. (2009b) suggested that $\mathrm{Si}$ variation is limited to massive or metal-poor GCs, based on the four GCs that show $\mathrm{Si}$ variation in their observations. This observational evidence agrees with our understanding of the nucleosynthesis reaction chains that are responsible for MPs, since more massive stars or more metal-poor stars tend to have higher reaction temperature, which is required for the $\mathrm{Mg}$-Al nuclear chain reaction and the associated Si leakage (Arnould et al. 1999). Including the GCs that show Si variation in their sample, NGC 2808, NGC 6388, NGC 6752, and NGC 7078 (M15), we found that NGC 5053 has the lowest cluster mass (Table 7). Depending on the mass estimation methods (McLaughlin \& van der Marel 2005, Baumgardt 2017), the masses of the clusters might be slightly different. However, we notice that the mass of NGC 5053 is constantly about one order of magnitude lower than other GCs. Therefore, this cluster likely has the lowest cluster mass among the GCs that have been identified to exhibit an observable Si spread until now.

Nevertheless, we caution that our sample size is limited. Ten stars are a reasonably statistically significant sample, but there is still a small chance that we may be missing the strong $\mathrm{Mg}$-depleted stars. Besides the ten stars that we study in this work, the CMD of NGC 5053 (Figure 2) indicates that another $\sim 10$ bright, RVidentified cluster members (B15) may be observable with the APOGEE-2 spectrograph (without considering fiber 
Table 7

Basic Parameters for GCs in this Paper.

\begin{tabular}{|c|c|c|c|c|c|}
\hline ID & Name & {$[\mathrm{Fe} / \mathrm{H}]^{a}$} & $\overline{\delta_{[\mathrm{Fe} / \mathrm{H}]}^{a}}$ & $\log$ Mass1 $^{\mathrm{b}}\left(\mathrm{M}_{\odot}\right)$ & $\operatorname{Mass}^{c}\left(\mathrm{M}_{\odot}\right)$ \\
\hline NGC 5053 & & -2.30 & 0.08 & $4.81 \pm 0.01$ & $5.37 \pm 1.32 \times 10^{4}$ \\
\hline NGC 7078 & M15 & -2.33 & 0.02 & & $5.01 \pm 0.06 \times 10^{5}$ \\
\hline NGC 6341 & M92 & -2.35 & 0.05 & $5.45 \pm 0.01$ & $3.05 \pm 0.04 \times 10^{5}$ \\
\hline NGC 5024 & M53 & -2.06 & 0.09 & $5.66 \pm 0.01$ & $3.83 \pm 0.51 \times 10^{5}$ \\
\hline NGC 2808 & & -1.18 & 0.04 & $5.93 \pm 0.01$ & $8.29 \pm 0.06 \times 10^{5}$ \\
\hline NGC 6388 & & -0.45 & 0.04 & $6.05 \pm 0.05$ & $1.24 \pm 0.01 \times 10^{6}$ \\
\hline NGC 6752 & & -1.55 & 0.01 & 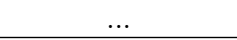 & $2.34 \pm 0.04 \times 10^{5}$ \\
\hline \begin{tabular}{l|l} 
From & Carre \\
From & McLa \\
From & Baum
\end{tabular} & $\frac{\operatorname{ta} \text { et al. }}{\text { gahlin } \frac{\alpha}{20}}$ & $\begin{array}{l}(2009 a) \\
\text { van der I } \\
17) .\end{array}$ & 212000 & & \\
\hline
\end{tabular}

collision). Since the $\mathrm{Mg}, \mathrm{Al}$, and $\mathrm{Si}$ lines are more difficult to be detected in the optical for metal-poor stars (Sbordone et al.2015), NIR high-resolution spectroscopy, e.g., APOGEE-2, is more appropriate.

\section{SUMMARY}

Driven by the desire to understand multiple populations in the oldest, most metal-poor stellar systems in the nearby Universe, we have carefully studied the orbit and chemical abundances of one of the most metal-poor GCs, NGC 5053. Given the similar location and RV between NGC 5053 and one of the Sgr arms, NGC 5053 has been speculated to be associated with Sgr. However, using our dynamical code GravPot16, we argue that a physical connection between Sgr and NGC 5053 is unlikely.

We have identified 10 cluster members in the APOGEE DR14 using their spatial location, RV, $[\mathrm{Fe} / \mathrm{H}]$, and CMD location. We detected strong $\mathrm{Mg}$, Al, and $\mathrm{Si}$ spectral lines in the APOGEE spectra, which are otherwise difficult to see in optical spectra. We manually analyzed the spectra with three different combinations of stellar parameters and codes. We proved that the large Al variation and the substantial Si spread are independent of the adopted measured method. Along with NGC 5053, metal-poor GCs exhibit different $\mathrm{Mg}, \mathrm{Al}$, and $\mathrm{Si}$ variations, which agrees with the claim that metallicity is not the only parameter that is fundamental to the MP phenomenon. Parameters such as cluster mass, must also play important roles. Interestingly, NGC 5053 has the lowest cluster mass among all the GCs that are currently found to exhibit Si variations. This may be the lower mass limit to generate an observable Si spread.

\section{ACKNOWLEDGMENTS}

We thank the anonymous referee for insightful comments. BT, JGFT, DG, and SV gratefully acknowledge support from the Chilean BASAL Centro de Excelencia en Astrofísica y Tecnologías Afines (CATA) grant PFB-06/2007. BT acknowledges support from the onehundred-talent project of Sun Yat-Sen University. SV gratefully acknowledges the support provided by Fondecyt reg. n. 1170518. OZ, TM, DAGH, and FD acknowledge support provided by the Spanish Ministry of Economy and Competitiveness (MINECO) under grant AYA2014-58082-P. DAGH was also funded by the Ramón y Cajal fellowship number RYC-2013-14182. SzM has been supported by the Premium Postdoctoral Research Program of the Hungarian Academy of Sciences, and by the Hungarian NKFI Grants K-119517 of the Hungarian National Research, Development and Innovation Office. TCB acknowledges partial support from grant PHY 14-30152; Physics Frontier Center/JINA Center for the Evolution of the Elements (JINA-CEE), awarded by the US National Science Foundation. STS acknowledges support by NASA through grants GO-14235 from the Space Telescope Science Institute (STScI), which is operated by the Association of Universities for Research in Astronomy (AURA), Inc., under NASA contract NAS5-26555. Funding for the GravPot16 software has been provided by the Centre national d'etudes spatiale (CNES) through grant 0101973 and UTINAM Institute of the Université de Franche-Comté, supported by the Région de FrancheComté and Institut des Sciences de l'Univers (INSU). Monte Carlo simulations have been executed on computers from the Utinam Institute of the Université de Franche-Comté, supported by the Région de FrancheComté and Institut des Sciences de l'Univers (INSU).

Funding for the Sloan Digital Sky Survey IV has been provided by the Alfred P. Sloan Foundation, the U.S. Department of Energy Office of Science, and the Participating Institutions. SDSS- IV acknowledges support and resources from the Center for High-Performance Computing at the University of Utah. The SDSS web site is www.sdss.org.

SDSS-IV is managed by the Astrophysical Research Consortium for the Participating Institutions of the SDSS Collaboration including the Brazilian Participation Group, the Carnegie Institution for Science, Carnegie Mellon University, the Chilean Participation Group, the French Participation Group, HarvardSmithsonian Center for Astrophysics, Instituto de Astrofîsica de Canarias, The Johns Hopkins University, Kavli Institute for the Physics and Mathematics of the Universe (IPMU) / University of Tokyo, Lawrence Berkeley National Laboratory, Leibniz Institut für Astrophysik Potsdam (AIP), Max-Planck-Institut für Astronomie (MPIA Heidelberg), Max-Planck-Institut für Astrophysik (MPA Garching), Max-Planck-Institut für Extraterrestrische Physik (MPE), National Astronomical Observatory of China, New Mexico State University, New York University, University of Notre Dame, Observatório Nacional / MCTI, The Ohio State University, Pennsylvania State University, Shanghai Astronomical Observatory, United Kingdom Participation Group, Universidad Nacional Autónoma de México, University of Arizona, University of Colorado Boulder, University of Oxford, University of Portsmouth, University of Utah, 
University of Virginia, University of Washington, University of Wisconsin, Vanderbilt University, and Yale Uni- versity.

\section{APPENDIX}

\section{GALACTIC MODEL}

For the Galactic model we employ a 3D steady-state gravitational potential of the Galaxy, assumed to be made up of the superposition of many composite stellar populations belonging to the thin disc, the thick disk, the Interstellar Matter (ISM), the stellar and dark matter halo, and a rotating Galactic bar component, that fit fairly well the structural and dynamical parameters of the Milky Way. The mass density distribution of each stellar and non-stellar component is described in detail in Robin et al. (2003, 2012, 2014) and the consequent semianalytic Milky Way potential shape will be fully described in a forthcoming paper (Fernández-Trincado et al. 2017, in preparation). Here, we present a summary of the main structural parameters of our 3D steady-state potential model; which have been adopted in a score of papers (see e.g., Fernández-Trincado et al. 2016, 2017a c b Abolfathi et al. 2017; Recio-Blanco et al. 2017, Anders et al. 2017; Libralato et al. 2018).

The model is made up of the sum of an axisymmetric background potential and a non-axisymmetric one. The axisymmetric potential is dominated by the superposition of homogenous oblate spheroids, which approximate the density models of Einasto (1979) thin disks (see e.g., Robin et al. 2003), while the ISM and the thick disks are the analytic expressions as introduced in Smith et al. (2015), which approximate the density profiles in Robin et al. (2003, 2014). The non-axisymmetric part includes a rotating bar, where the corresponding potential model consists of triaxial inhomogeneous ellipsoids similar to Pichardo et al. (2004), which approximate the dimensions and density model of Robin et al. (2012). In this work, the overall Galactic potential has been scaled to the Sun's Galactocentric distance, $8 \mathrm{kpc}$, and the local rotation velocity of $244.5 \mathrm{~km} \mathrm{~s}^{-1}$ (e.g., Sofue 2015). The adopted values for the Sun's orbital velocity vector are $\left[U_{\odot}, V_{\odot}, W_{\odot}\right]=[13.12 \pm 1.47,0.92 \pm 0.29,7.03 \pm 0.18] \mathrm{km} \mathrm{s}^{-1}$ (e.g., Robin et al. 2017). For reference, we have adopted a righthanded coordinate system for $U, V$ and $W$, so that they are positive in the directions of the Galactic center, Galactic rotation, and North Galactic Pole, respectively.

For the structural parameters of the bar potential we adopted the dynamical constraints from 3D test particle simulations evolved in the 3D steady-state Milky Way potential above mentioned (Fernández-Trincado|2017; FernándezTrincado et al. 2017b), which is able to reproduce the velocity rotation curve of stars in the bulge at different Galactic longitude and latitudes, as illustrated in Figure 12. For our computations, we have considered the total mass for the bar, $1.1 \times 10^{10} \mathrm{M}_{\odot}$, the present-day orientation of the major axis of the Galactic bar, $\phi=20^{\circ}$, and a pattern speed, $\Omega_{\mathrm{B}}=35 \mathrm{~km} \mathrm{~s}^{-1} \mathrm{kpc}^{-1}$. These values are consistent with the recent estimates from literature (see Portail et al. 2015, for instance).

\section{REFERENCES}

Abolfathi, B., Aguado, D. S., Aguilar, G., et al. 2017, ArXiv e-prints, arXiv:1707.09322

Anders, F., Queiroz, A. B., Chiappini, C., et al. 2017, ArXiv e-prints, arXiv:1708.09319

Arellano Ferro, A., Giridhar, S., \& Bramich, D. M. 2010, MNRAS, 402, 226

Arnould, M., Goriely, S., \& Jorissen, A. 1999, A\&A, 347, 572

Asplund, M., Grevesse, N., \& Sauval, A. J. 2005, in Astronomical Society of the Pacific Conference Series, Vol. 336, Cosmic Abundances as Records of Stellar Evolution and

Nucleosynthesis, ed. T. G. Barnes, III \& F. N. Bash, 25

Baumgardt, H. 2017, MNRAS, 464, 2174

Bellazzini, M., Ferraro, F. R., \& Ibata, R. 2003, AJ, 125, 188

Boberg, O. M., Friel, E. D., \& Vesperini, E. 2015, ApJ, 804, 109

Carretta, E., Bragaglia, A., Gratton, R., D'Orazi, V., \& Lucatello, S. 2009a, A\&A, 508, 695

Carretta, E., Bragaglia, A., Gratton, R., \& Lucatello, S. 2009b, A\&A, 505, 139

Carretta, E., Bragaglia, A., Gratton, R. G., et al. 2014, A\&A, 561, A 87

-. 2010a, A\&A, 516, A55

Carretta, E., Bragaglia, A., Lucatello, S., et al. 2017, A\&A, 600, A118

Carretta, E., Bragaglia, A., Gratton, R. G., et al. 2010b, A\&A, 520, A95

Chun, S.-H., Kim, J.-W., Sohn, S. T., et al. 2010, AJ, 139, 606

Cohen, J. G. 2004, AJ, 127, 1545

D'Antona, F., Vesperini, E., D'Ercole, A., et al. 2016, MNRAS, 458,2122

Dotter, A., Chaboyer, B., Jevremović, D., et al. 2008, ApJS, 178, 89

Einasto, J. 1979, in IAU Symposium, Vol. 84, The Large-Scale Characteristics of the Galaxy, ed. W. B. Burton, 451-458

Eisenstein, D. J., Weinberg, D. H., Agol, E., et al. 2011, AJ, 142, 72

Fernández-Alvar, E., Carigi, L., Schuster, W. J., et al. 2018, ApJ, 852,50

Fernández-Trincado, J. G. 2017, Université Bourgogne

Franche-Comté Phd Thesis, 1, 174
Fernández-Trincado, J. G., Geisler, D., Moreno, E., et al. 2017a, ArXiv e-prints, arXiv:1710.07433

Fernández-Trincado, J. G., Robin, A. C., Moreno, E., Pérez-Villegas, A., \& Pichardo, B. 2017b, ArXiv e-prints, arXiv: 1708.05742

Fernández-Trincado, J. G., Robin, A. C., Moreno, E., et al. 2016, ApJ, 833, 132

Fernández-Trincado, J. G., Zamora, O., García-Hernández, D. A., et al. 2017c, ApJ, 846, L2

García-Hernández, D. A., Mészáros, S., Monelli, M., et al. 2015, ApJ, 815, L4

García Pérez, A. E., Allende Prieto, C., Holtzman, J. A., et al. 2016, AJ, 151, 144

González Hernández, J. I. \& Bonifacio, P. 2009, A\&A, 497, 497

Gunn, J. E., Siegmund, W. A., Mannery, E. J., et al. 2006, AJ, 131,2332

Harris, W. E. 1996, AJ, 112, 1487

Hasselquist, S., Shetrone, M., Smith, V., et al. 2017, ApJ, 845, 162

Hayes, C. R., Majewski, S. R., Shetrone, M., et al. 2018, ApJ, 852,49

Holtzman, J. A., Shetrone, M., Johnson, J. A., et al. 2015, AJ, 150,148

Howard, C. D., Rich, R. M., Reitzel, D. B., et al. 2008, ApJ, 688, 1060

Kharchenko, N. V., Piskunov, A. E., Schilbach, E., Röser, S., \& Scholz, R.-D. 2013, A\&A, 558, A53

Kunder, A., \& Chaboyer, B. 2009, AJ, 137, 4478

Kunder, A., Koch, A., Rich, R. M., et al. 2012, AJ, 143, 57

Lauchner, A., Powell, Jr., W. L., \& Wilhelm, R. 2006, ApJ, 651, L33

Law, D. R., \& Majewski, S. R. 2010, ApJ, 718, 1128

Libralato, M., Bellini, A., Bedin, L. R., et al. 2018, ArXiv e-prints, arXiv:1801.01502

Majewski, S. R., Schiavon, R. P., Frinchaboy, P. M., et al. 2017, AJ, 154, 94

Masseron, T., Merle, T., \& Hawkins, K. 2016, BACCHUS: Brussels Automatic Code for Characterizing High accUracy Spectra, Astrophysics Source Code Library, , , ascl:1605.004

McLaughlin, D. E., \& van der Marel, R. P. 2005, ApJS, 161, 304

Mészáros, S., Martell, S. L., Shetrone, M., et al. 2015, AJ, 149, 153

Milone, A. P., Marino, A. F., Piotto, G., et al. 2015, ApJ, 808, 51 

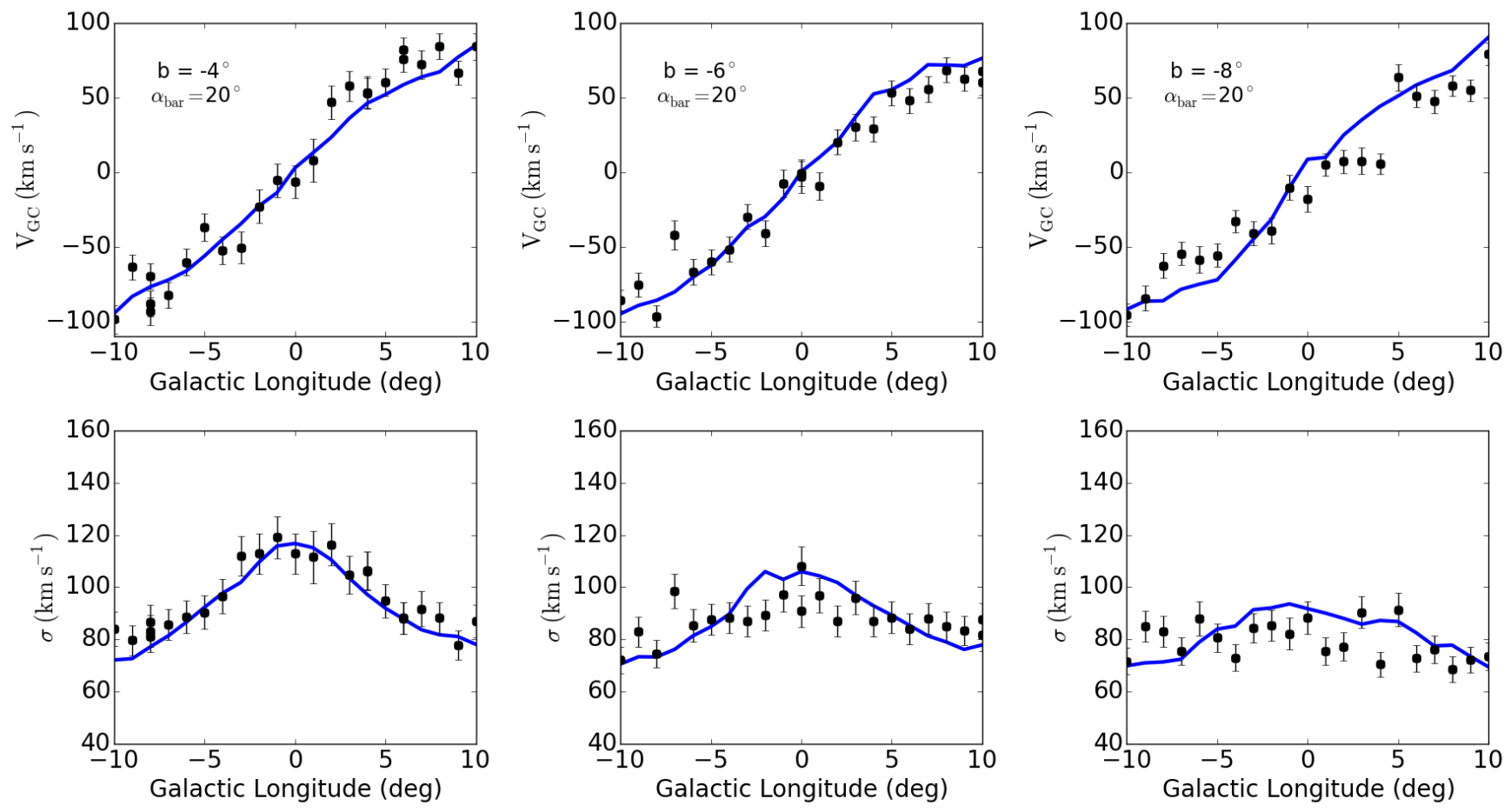

Figure 12. Rotation curve (blue) of a boxy bulge from 3D test particle simulations evolved in 3D steady-state potential model ( GravPot16) compared to BRAVA (black) data (Howard et al. 2008 Kunder et al. 2012). Taken from Figure 5.12 in Fernández-Trincado (2017)

Moreno, E., Pichardo, B., \& Schuster, W. J. 2015, MNRAS, 451, 705

Mottini, M., Wallerstein, G., \& McWilliam, A. 2008, AJ, 136, 614

Nidever, D. L., Holtzman, J. A., Allende Prieto, C., et al. 2015, AJ, 150,173

Palma, C., Majewski, S. R., \& Johnston, K. V. 2002, ApJ, 564, 736

Pancino, E., Romano, D., Tang, B., et al. 2017, A\&A, 601, A112

Pichardo, B., Martos, M., \& Moreno, E. 2004, ApJ, 609, 144

Piotto, G., Milone, A. P., Bedin, L. R., et al. 2015, AJ, 149, 91

Plez, B. 2012, Turbospectrum: Code for spectral synthesis, Astrophysics Source Code Library, , , ascl:1205.004

Portail, M., Wegg, C., Gerhard, O., \& Martinez-Valpuesta, I. 2015, MNRAS, 448, 713

Prantzos, N., Charbonnel, C., \& Iliadis, C. 2007, A\&A, 470, 179

Pryor, C., Piatek, S., \& Olszewski, E. W. 2010, AJ, 139, 839

Recio-Blanco, A., Rojas-Arriagada, A., de Laverny, P., et al. 2017, A\&A, 602, L14

Robin, A. C., Bienaymé, O., Fernández-Trincado, J. G., \& Reylé, C. 2017, A\&A, 605, A1

Robin, A. C., Marshall, D. J., Schultheis, M., \& Reylé, C. 2012, A\&A, 538, A106

Robin, A. C., Reylé, C., Derrière, S., \& Picaud, S. 2003, A\&A, 409, 523

Robin, A. C., Reylé, C., Fliri, J., et al. 2014, A\&A, 569, A13

Sarajedini, A., \& Milone, A. A. E. 1995, AJ, 109, 269

Sbordone, L., Bonifacio, P., Buonanno, R., et al. 2007, A\&A, 465, 815
Sbordone, L., Monaco, L., Moni Bidin, C., et al. 2015, A\&A, 579, A104

Schiavon, R. P., Johnson, J. A., Frinchaboy, P. M., et al. 2017, MNRAS, 466, 1010

Schlafly, E. F., \& Finkbeiner, D. P. 2011, ApJ, 737, 103

Shetrone, M., Bizyaev, D., Lawler, J. E., et al. 2015, ApJS, 221, 24

Smith, R., Flynn, C., Candlish, G. N., Fellhauer, M., \& Gibson, B. K. 2015, MNRAS, 448, 2934

Sofue, Y. 2015, PASJ, 67, 75

Sohn, S. T., Anderson, J., \& van der Marel, R. P. 2012, ApJ, 753, 7

Sohn, S. T., Besla, G., van der Marel, R. P., et al. 2013, ApJ, 768, 139

Sohn, S. T., Patel, E., Besla, G., et al. 2017, ApJ, 849, 93

Souto, D., Cunha, K., Smith, V., et al. 2016, ApJ, 830, 35

Tang, B., Cohen, R. E., Geisler, D., et al. 2017, MNRAS, 465, 19

Ventura, P., D'Antona, F., Di Criscienzo, M., et al. 2012, ApJ, 761, L30

Zamora, O., García-Hernández, D. A., Allende Prieto, C., et al. 2015, AJ, 149, 181

Zasowski, G., Cohen, R. E., Chojnowski, S. D., et al. 2017, ArXiv e-prints, arXiv:1708.00155 